\title{
The Importance of Mathematical Models to Explore the Effects of Marijuana and Other Plant Based Products on Learning and Memory
}

\section{Charles I. Abramson, Sarah Markland, Igor I. Stepanov}

Laboratory of Comparative Psychology and Behavioral Biology, Departments of Psychology and Integrative Biology, Oklahoma State University, Stillwater, OK, USA

Correspondence to: Charles I. Abramson, charles.abramson@okstate.edu

Keywords: Learning, Memory, Marijuana, Mathematical Models, Plant Based Products

Received: April 2, $2019 \quad$ Accepted: May 27, $2019 \quad$ Published: May 30, 2019

Copyright $\odot 2019$ by authors and Scientific Research Publishing Inc.

This work is licensed under the Creative Commons Attribution International License (CC BY 4.0).

http://creativecommons.org/licenses/by/4.0/

(c) (i) Open Access

\section{ABSTRACT}

This article describes the use of the first order system transfer function for learning and memory studies involving consumption of marijuana and other plant based products. We provide detailed instructions on how the model can be used to analyze the performance of individual participants using a memory test developed by the senior authors. The importance of identifying possible learning and memory deficits of marijuana is paramount due to the growing number of states in the U.S. legalizing marijuana use for medicinal and recreational purposes. The model can also be extended to other plant based products purported to improve memory. While this article does not study the effect of marijuana, we provide details on how it can be used by illustrating its application on individuals consuming an amphetamine-like psychostimulant drug using our own memory test.

\section{INTRODUCTION}

The purpose of this article is two-fold. First, we advocate the use of mathematical models to characterize the learning process for researchers interested in exploring the effects of marijuana on learning and memory. While articles contain the results of statistical tests, to our knowledge no previous article has promoted the use of mathematical models to characterize potential learning and memory effects either on marijuana or other plant based products purporting to improve memory. Mathematical models have much to recommend them for the study of marijuana and other plant based products. Such models can easily summarize large amounts of data, direct research, stimulate the development of theory, and guide therapeutic intervention. They can also be used to test claims made by advocates of plant based products that such products improve learning and memory.

Second, we advocate the use of the first order system transfer function. We have used this model in a wide variety of studies ranging from the assessment of memory in drug addicts and alcoholics [1] to the 
detection of memory impairment in individuals suffering from either Type-2 diabetes mellitus [2] or multiple sclerosis [3]. The model has also been applied to both the child and adult versions of the California Verbal Learning Test $[4,5]$. Moreover, we have used the model to detect subspecies differences in the maze performance of rats [6] and to characterize the learning deficits in honey bees exposed to agrochemicals $[7,8]$.

The remainder of this article describes the mathematical foundation of our model and finishes with an example of how the model is used in practice. We would like to note that the material is presented in detail to provide the reader with a full description of how the model is used. The raw learning data, along with the calculated coefficients, are provided for each individual in table form. Figures are also provided describing the quality of the model fit with actual data. The senior authors (IS and CA) would be glad to assist any researcher in applying the model.

\section{DESCRIPTION OF THE MODEL}

The mathematical background of the application of the transfer function of the first order linear system in response to a stepwise input action for assessment of the learning curve was provided elsewhere [6]. During a free recall memory test (such as the CVLT-II, Pearson, Bloomington MN), presentation of a list of words acts as a stepwise input signal that begins to act upon a participant under testing for the first time on the first trial. The senior authors adopted the first order transfer function for the assessment of the learning curve in the form $Y=B_{3} \mathrm{e}^{-B_{2}(X-1)}+B_{4}\left(1-\mathrm{e}^{-B_{2}(X-1)}\right)$.

If researchers are interested in using the California Verbal Learning Test (CVLT, Pearson, Bloomington MN) to study marijuana or other plant based products, $X$ is the trial number and $Y$ is the number of correctly recalled words without repetitions. The parameters are: $B_{2}$-the learning rate; $B_{4}$-the asymptotic value of recalled words at $X=$ Infinity; $B_{3}$-the number of correctly recalled words on the first trial (i.e. $B_{3}$ $=Y$ at $X=1)$. The same nomenclature is used if a researcher wishes to use the memory test developed by the senior authors.

Use of the independent variable in the form of $(x-1)$ means that the first trial, i.e. at $x=1$, assesses auditory attention span [9] or in our terminology, the background readiness to learn. Learning itself begins when the list of words is presented repeatedly, Trial 2 being the first repetition. In accordance with the definition of the transfer function, the rate of achievement of the asymptotic value is named "the time constant $(\tau)$ ". The time constant reveals how much time (after Trial 1 ) is necessary for achievement of $63 \%$ of the difference between the initial and asymptotic levels of the output signal. Thus, regarding learning of target words, $1 / B_{2}$ is the number of trials after Trial 1 needed to reach $63 \%$ from the difference between the initial $\left(B_{3}\right)$ and asymptotic $\left(B_{4}\right)$ values of correctly recalled words.

Comparison of the CVLT/CVLT-II standard measures that are in use for List A versus the model's coefficients shows that coefficient $B_{3}$ is very close to the level of correct recall on Trial 1 -the standard measure used in the CVLT. However, coefficients $B_{2}$ and $B_{4}$ do not have an analogue among other CVLT standard measures. When applying the model to human learning, the coefficient $B_{3}$ can be considered as the short-term memory estimator and the coefficient $B_{4}$ is the long-term memory estimator. SPSS Statistics 17.0 and Mathematica 6 were used for all statistical calculations and graph drawing for this article.

\section{THE SENIOR AUTHORS' FREE RECALL MEMORY TEST (IS \& CA)}

Previously, modeling of the learning curves was applied to raw learning data from CVLT/CVLT-II, List A, and CVLT-C. However, the use of the mathematical model imposes certain additional requirements for carrying out the free recall memory test in order to obtain accurate (not distorted) information about the memory state of the examinee. In connection with the above, we identified a need to develop our own version of the free recall memory test that meets all of the necessary requirements to ensure the effectiveness of mathematical modeling.

The first requirement concerns the size of the word list. How should the optimal number of words for 
the list be chosen? We found that healthy females could recall up to 12 - 13 words from a list. Therefore, the list must include at least 13 words to correctly assess levels of short-term memory. This means that ten words, which are used in some tests, may not be enough to assess short-term memory. On the other hand, what size of a list should be used for correct assessment of long-term memory and to prevent the so-called "ceiling effect"? Obviously, the quantity of words in a list should exceed 13 words for the correct assessment of long-term memory. The CVLT/CVLT-II, List A includes 16 words, but the authors of the test did not explain their choice. We might consider the explanation to be that they used four semantic categories with four words in each category. Similarly, Zimprich and Rast [10] used a list of 27 meaningful, but unrelated, two to three syllable German words.

From our point of view, the size of a list depends on the group of people for whom the test is intended. If the task is to study how many words healthy persons can learn and recall, a list can be increased up to 27 words. If the memory test is developed for the assessment of marijuana users with initial signs of memory impairment, from our point of view, 16 - 20 words is enough. If a list exceeds 20 words, its use in marijuana users might lead to fatigue and even have some neurological consequences. Thus, the senior authors constructed a word list consisting of 16 words, taken from four semantic categories: vegetables, animals, ways of traveling, and furniture, in such a way that no two words are from the same semantic category. Moreover, no two words from the same semantic category were placed side by side.

The second requirement deals with deciding on the optimal number of trials. The CVLT/CVLT-II uses five trials. However, many individuals do not have time to reach their asymptotic level of correctly recalled words with five presentations of a list of words. As a result, coefficient B4 returns overestimated values, which requires an additional correction. Alternatively, some tests use too many trials, for example, ten trials are used in Luria's memory test. The large number of test trials can produce fatigue even without exposure to marijuana [11]. This is why the senior authors selected six trials, as this is sufficient to assess the asymptotic level of B4 value without producing fatigue.

The third requirement deals with the order of words during the second and subsequent presentation of the list. In CVLT/CVLT-II, the order of words in List A is constant during each trial to assess primacy/recency recall. However, from a modeling point of view, it is desirable to let the participant learn each word from the list equally. That is why the order of words in the list is changed on each trial. Note that Zimprich and Rast [10] used the same approach. In principle, we moved each word from its initial position in the list to an opposite position, for example, from the beginning or the end region of the list to the middle region and vice versa.

The fourth requirement deals with so-called "item-specific" practice that refers to the learning of actual content (e.g., a word list) from one administration of the test to the next [12]. The item-specific practice can be mitigated via the use of alternate test forms with different lists of words. That is why six lists of 16 words were constructed from the same semantic categories. A total of six lists allows avoiding "item-specific" practice during six test/retest administrations for each participant.

The fifth requirement deals with testing/retesting of the participant. The learning curves of the same participant are never identical during retesting. There are at least two possible sources of such variability. First, as with most cognitive tests, the participant is apt to develop test-taking strategies with repeated exposures to the same procedure. Benedict \& Zgaljardic [13] refer to this as "test-specific" practice. Second, the participant's state of health might be changed due to any external or internal factors acting before or during test performance, such as exposure to marijuana. Thus, it is advisable to conduct several repeated tests for each participant. We advocate repeating the test three times.

\section{CALCULATING MEMORY LEVELS: INDIVIDUALS EXPOSED TO AMPHETAMINE-LIKE PSYCHOSTIMULANT DRUGS}

Mathematical modeling of the learning curve offers a quantitative description of learning and memory abilities when a participant is exposed to marijuana or consumes a plant based product purported to improve learning and memory. Moreover, values of the model's coefficients B3 and B4 give the opportu- 
nity to characterize short- and long-term memory status quantitatively. For this purpose, it is necessary to separate the initial set of B3 and B4 values into disjointed subsets.

When the model was applied to patients with multiple sclerosis, about half of the patients were shown to exhibit cognitive impairment [14]. Thus, when working with patients suffering from multiple sclerosis, it is possible to obtain a spectrum from "normal memory status" to "severely impaired". The multiple sclerosis group included 365 patients (266 females and 99 males) with clinically defined multiple sclerosis and was tested with the CVLT-II [14]. The raw learning data (the number of correctly recalled words from List A) were modeled and the sets of B3 and B4 values were used for further analysis [3]. Female and male sets of B3 values, as well as B4 values, were analyzed separately because it was shown that females scored significantly higher than males on the CVLT [15].

SPSS 17 was used for cluster and discriminant analysis. Initial values of B3 or B4 were classified based on hierarchical cluster analysis. This procedure identifies relatively homogeneous groups of B3 or B4 values, using an algorithm that starts with each value in a separate cluster, and combines clusters until only one is left. At each step, the two clusters separated by the shortest distance are combined. After separating raw coefficient values into six clusters of B3 values or six clusters of B4 values, discriminant analysis is applied to the clusters. Discriminant analysis creates a linear function of B3 or B4 that provides the best discrimination between two samples, in particular, between neighboring clusters. With discriminant analysis, it is possible to calculate cut-off values B3 and B4 for assigning the obtained B3 or B4 value to one or another memory state level. In other words, the cut-off values allow the determination of to what extent the short- and long-term memory is impaired.

\section{SUBJECTS}

The data were gathered at the Institute for Experimental Medicine, St. Petersburg Russia between January and April, 2013. The research was conducted in accordance with the National Standard of the Russian Federation "Good Clinical Practice" with the permission of the Institutional Board on Biomedical Ethics of the Institute for Experimental Medicine. Participants were recruited from the Institute's clinic who were undergoing drug rehabilitation and wanted to continue their treatment. Healthy volunteers were recruited from the surrounding area. Two groups were used. The first group was 15 healthy volunteers and was comprised of seven females within the age range of $22-27$ years old and eight males within the age range of 23 - 31 years old. The second group was 15 drug addicts and was comprised of four females within the age range of 23 - 31 years old and 11 males within the age range of 22 - 44 years old who all underwent rehabilitation courses. The drug addicts used Amphetamine-like psychostimulant drugs.

Each participant was examined three times with an intertest interval of one to two days. Three different lists of words were used. The senior authors are grateful to Professor Sergey Tsikunov, Pavlov's Department of Physiology, Institute for Experimental Medicine, St. Petersburg, Russia who conducted clinical examinations of the subjects. Based on this assessment, participants were assigned either to the healthy volunteer group or the drug addicted group.

\section{RESULTS}

\section{Healthy participants, females}

Raw learning data are given in Table 1 and our model's coefficients for individual learning curves are given in Table 2 .

Figures below show three individual learning curves for each participant (see Figures 1-7).

It is seen that during the three tests, each participant recalled a different number of words on Trial 1 , but recalled practically all the words from the list on the later trials. Four participants (ID = C3, C8, C11, C12) monotonously increased Trial 1 values from test 1 to test 3 . This order was different in other participants (ID $=\mathrm{C} 4, \mathrm{C} 6, \mathrm{C} 14)$. Thus, in healthy females, averaging over three tests results in a more precise short-term memory assessment (coefficient B3).

Analysis of short-term memory levels is presented in Table 3 and long-term memory levels in Table 4. 
Table 1. Raw learning data for healthy group, females.

\begin{tabular}{cccccccc}
\hline ID & Test/retest & Trial 1 & Trial 2 & Trial 3 & Trial 4 & Trial 5 & Trial 6 \\
\hline & 1 & 10 & 13 & 16 & 16 & 16 & 16 \\
C3 & 2 & 12 & 14 & 15 & 15 & 16 & 16 \\
& 3 & 13 & 15 & 16 & 16 & 16 & 16
\end{tabular}

Mean \pm S.E.M. $11.67 \pm 0.88214 .00 \pm 0.57715 .67 \pm 0.33315 .67 \pm 0.33316 .00 \pm 0.00016 .00 \pm 0.000$

\begin{tabular}{|c|c|c|c|c|c|c|}
\hline & 1 & 10 & 13 & 14 & 16 & 16 \\
\hline & 2 & 12 & 14 & 16 & 16 & 16 \\
\hline & 3 & 9 & 11 & 15 & 14 & 15 \\
\hline
\end{tabular}

Mean \pm S.E.M. $10.33 \pm 0.88212 .67 \pm 0.88215 .00 \pm 0.577 \quad 15.33 \pm 0.66715 .67 \pm 0.33316 .00 \pm 0.000$

\begin{tabular}{|c|c|c|c|c|c|c|c|}
\hline & 1 & 11 & 13 & 15 & 16 & 16 & 16 \\
\hline \multirow{2}{*}{ C6 } & 2 & 10 & 11 & 13 & 14 & 16 & 16 \\
\hline & 3 & 12 & 14 & 14 & 15 & 16 & 16 \\
\hline
\end{tabular}

Mean \pm S.E.M. $11.33 \pm 0.88213 .33 \pm 0.66715 .00 \pm 1.00015 .00 \pm 1.00015 .67 \pm 0.33316 .00 \pm 0.000$

\begin{tabular}{|c|c|c|c|c|c|c|}
\hline 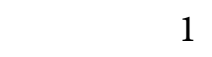 & 10 & 14 & 16 & 16 & 16 & 16 \\
\hline 2 & 11 & 12 & 13 & 13 & 15 & 16 \\
\hline 3 & 13 & 14 & 16 & 16 & 16 & 6 \\
\hline
\end{tabular}

Mean \pm S.E.M. $11.33 \pm 0.88213 .33 \pm 0.66715 .00 \pm 1.00015 .00 \pm 1.00015 .67 \pm 0.33316 .00 \pm 0.000$

\begin{tabular}{|c|c|c|c|c|c|}
\hline 1 & 8 & 10 & 12 & 13 & 15 \\
\hline 2 & 9 & 11 & 12 & 14 & 16 \\
\hline 3 & 11 & 13 & 15 & 16 & 16 \\
\hline
\end{tabular}

Mean \pm S.E.M. $\quad 9.33 \pm 0.88211 .33 \pm 0.88213 .00 \pm 1.00014 .33 \pm 0.88215 .67 \pm 0.33316 .00 \pm 0.000$

\begin{tabular}{|c|c|c|c|c|c|c|}
\hline 1 & 10 & 14 & 16 & 16 & 16 & 16 \\
\hline 2 & 11 & 14 & 15 & 16 & 15 & 16 \\
\hline 3 & 12 & 14 & 16 & 16 & 16 & 16 \\
\hline
\end{tabular}

Mean \pm S.E.M. $11.00 \pm 0.57714 .00 \pm 0.00015 .67 \pm 0.33316 .00 \pm 0.00015 .67 \pm 0.33316 .00 \pm 0.000$

\begin{tabular}{|c|c|c|c|c|c|}
\hline 1 & 7 & 10 & 13 & 16 & 16 \\
\hline 2 & 13 & 14 & 15 & 16 & 15 \\
\hline 3 & 10 & 12 & 14 & 16 & 16 \\
\hline
\end{tabular}

Mean \pm S.E.M. $10.00 \pm 1.73212 .00 \pm 1.15514 .00 \pm 0.57716 .00 \pm 0.00015 .67 \pm 0.33316 .00 \pm 0.000$ 
Table 2. Individual learning curves for healthy group, females.

\begin{tabular}{|c|c|c|c|c|c|}
\hline \multirow{2}{*}{ ID } & \multirow{2}{*}{ Test/retest } & \multicolumn{3}{|c|}{ Model's coefficients } & \multirow{2}{*}{$\mathrm{R}^{2}$} \\
\hline & & B2 & B3 & B4 & \\
\hline \multirow{4}{*}{$\mathrm{C} 3$} & 1 & $0.875 \pm 0.270$ & $9.871 \pm 0.614$ & $16.340 \pm 0.510$ & 0.964 \\
\hline & 2 & $0.583 \pm 0.172$ & $12.050 \pm 0.306$ & $16.177 \pm 0.412$ & 0.974 \\
\hline & 3 & $1.180 \pm 0.185$ & $12.981 \pm 0.152$ & $16.078 \pm 0.101$ & 0.990 \\
\hline & Averaged & $0.862 \pm 0.156$ & $11.627 \pm 0.250$ & $16.125 \pm 0.211$ & 0.987 \\
\hline \multirow{4}{*}{$\mathrm{C} 4$} & 1 & $0.576 \pm 0.160$ & $9.994 \pm 0.459$ & $16.602 \pm 0.629$ & 0.977 \\
\hline & 2 & $0.875 \pm 0.270$ & $11.914 \pm 0.409$ & $16.227 \pm 0.340$ & 0.964 \\
\hline & 3 & $0.513 \pm 0.309$ & $8.835 \pm 1.020$ & $16.283 \pm 1.661$ & 0.912 \\
\hline & Averaged & $0.626 \pm 0.134$ & $10.241 \pm 0.343$ & $16.280 \pm 0.419$ & 0.985 \\
\hline \multirow{4}{*}{ C6 } & 1 & $0.597 \pm 0.157$ & $10.872 \pm 0.381$ & $16.544 \pm 0.495$ & 0.979 \\
\hline & 2 & $0.356 \pm 0.283$ & $10.000 \pm 0.845$ & $16.835 \pm 2.553$ & 0.926 \\
\hline & 3 & $0.337 \pm 0.207$ & $12.000 \pm 0.448$ & $16.996 \pm 1.498$ & 0.943 \\
\hline & Averaged & $0.584 \pm 0.135$ & $11.316 \pm 0.283$ & $16.158 \pm 0.380$ & 0.984 \\
\hline \multirow{4}{*}{$\mathrm{C} 8$} & 1 & $1.180 \pm 0.185$ & $9.962 \pm 0.304$ & $16.155 \pm 0.202$ & 0.990 \\
\hline & 2 & $0.250 \pm 0.348$ & $11.000 \pm 0.799$ & $16.868 \pm 4.676$ & 0.877 \\
\hline & 3 & $0.689 \pm 0.348$ & $12.862 \pm 0.486$ & $16.299 \pm 0.524$ & 0.917 \\
\hline & Averaged & $0.584 \pm 0.135$ & $11.316 \pm 0.283$ & $16.158 \pm 0.380$ & 0.984 \\
\hline \multirow{4}{*}{$\mathrm{C} 11$} & 1 & $0.349 \pm 0.189$ & $8.000 \pm 0.727$ & $16.821 \pm 2.276$ & 0.962 \\
\hline & 2 & $0.377 \pm 0.256$ & $9.000 \pm 0.889$ & $16.852 \pm 2.425$ & 0.936 \\
\hline & 3 & $0.597 \pm 0.157$ & $10.872 \pm 0.381$ & $16.544 \pm 0.495$ & 0.979 \\
\hline & Averaged & $0.392 \pm 0.129$ & $9.33 \pm 0.43$ & $16.84 \pm 1.09$ & 0.982 \\
\hline \multirow{4}{*}{$\mathrm{C} 12$} & 1 & $1.180 \pm 0.185$ & $9.962 \pm 0.304$ & $16.155 \pm 0.202$ & 0.990 \\
\hline & 2 & $1.009 \pm 0.340$ & $10.995 \pm 0.503$ & $15.742 \pm 0.370$ & 0.956 \\
\hline & 3 & $0.875 \pm 0.270$ & $11.914 \pm 0.409$ & $16.227 \pm 0.340$ & 0.964 \\
\hline & Averaged & $1.029 \pm 0.185$ & $10.960 \pm 0.287$ & $16.037 \pm 0.208$ & 0.987 \\
\hline \multirow{4}{*}{ C14 } & 1 & $0.553 \pm 0.224$ & $7.000 \pm 0.962$ & $16.823 \pm 1.398$ & 0.960 \\
\hline & 2 & $0.547 \pm 0.393$ & $12.932 \pm 0.529$ & $16.002 \pm 0.781$ & 0.871 \\
\hline & 3 & $0.486 \pm 0.193$ & $10.000 \pm 0.590$ & $16.868 \pm 1.045$ & 0.966 \\
\hline & Averaged & $0.459 \pm 0.178$ & $9.818 \pm 0.569$ & $16.979 \pm 1.107$ & 0.966 \\
\hline
\end{tabular}




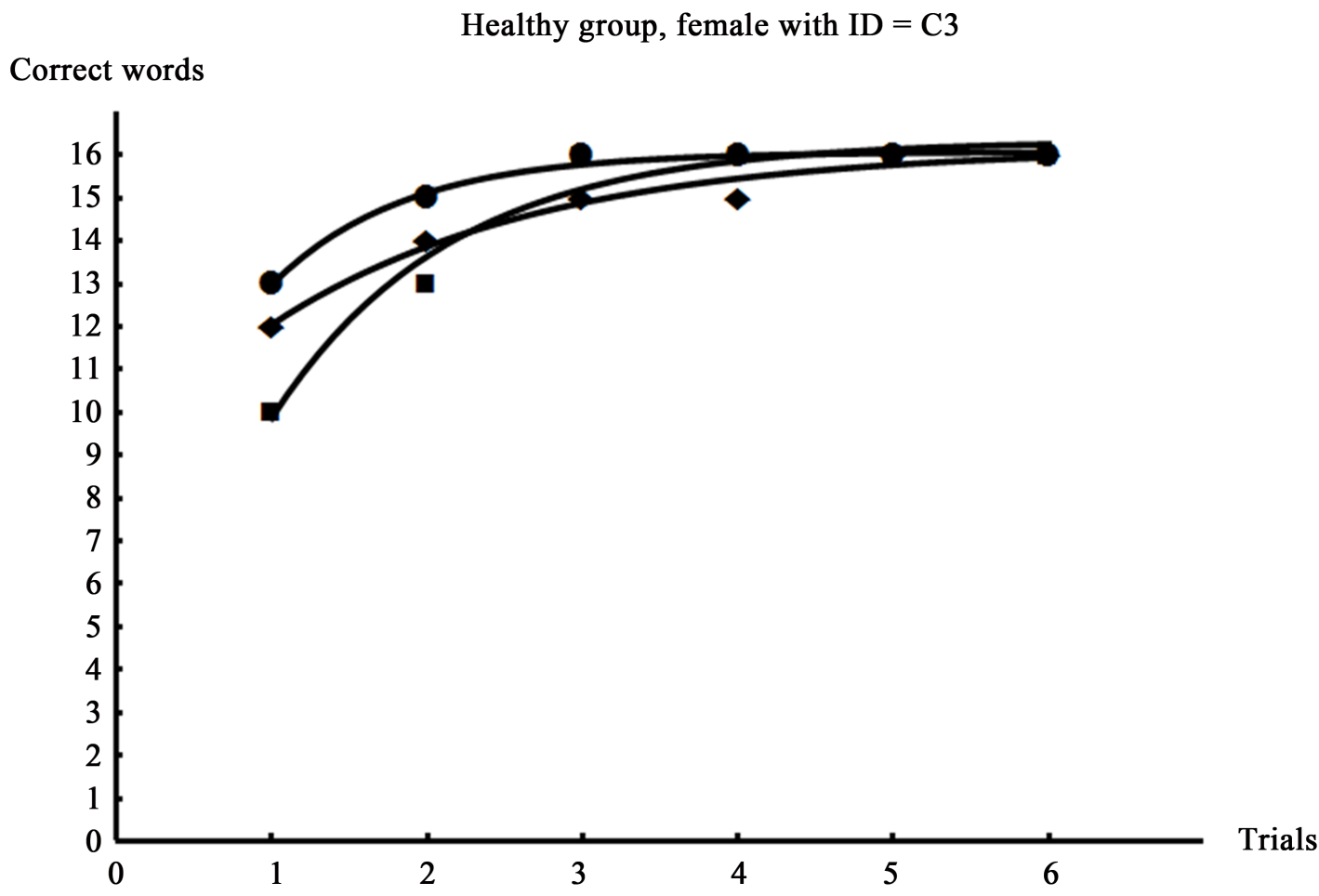

Figure 1 . Healthy group, female with ID $=\mathrm{C} 3$. Squares-the first test, diamonds-the second test, circles-the third test.

Healthy group, female with ID $=\mathrm{C} 4$

\section{Correct words}

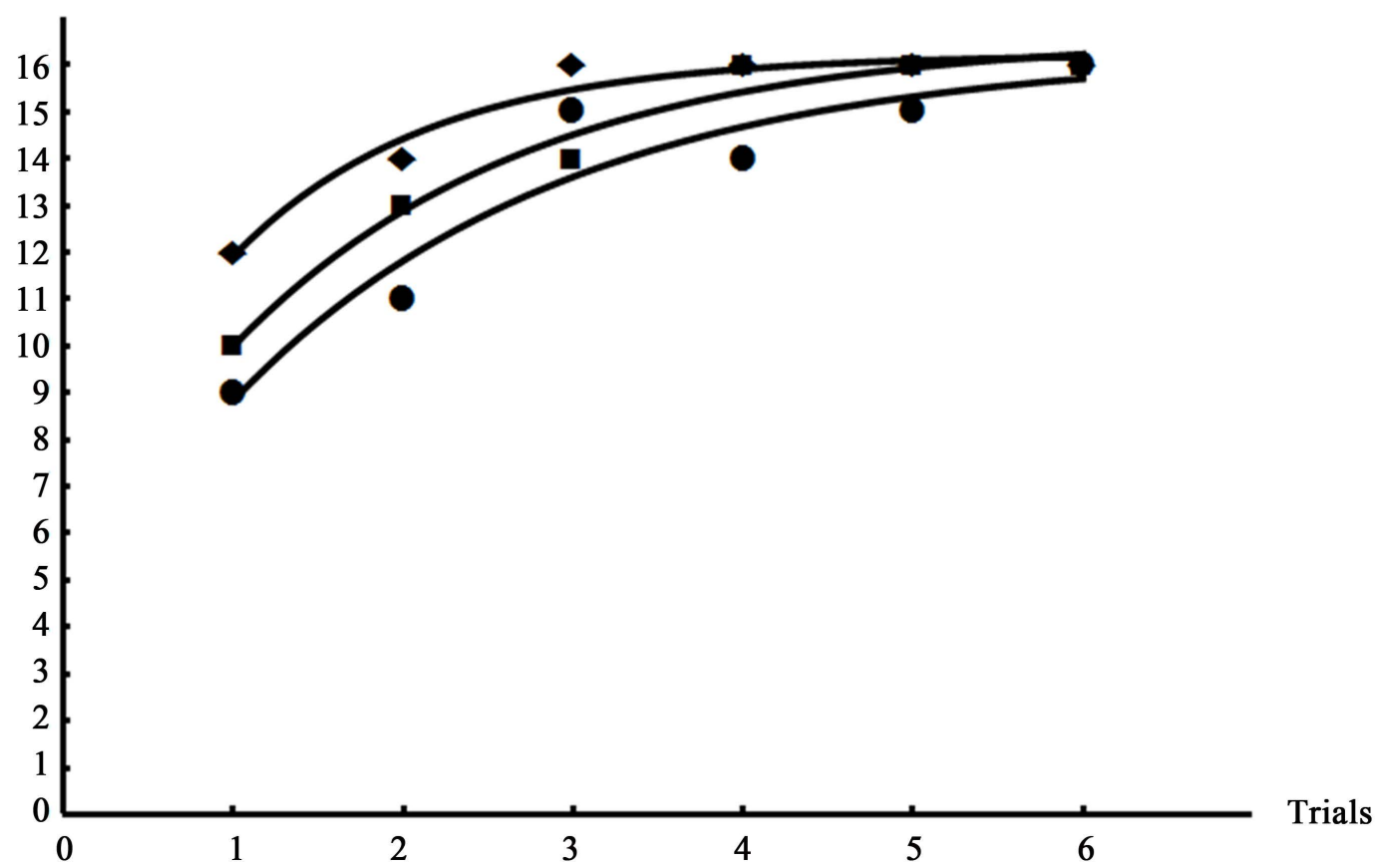

Figure 2. Healthy group, female with ID $=\mathrm{C} 4$. Squares-the first test, diamonds-the second test, circles-the third test. 


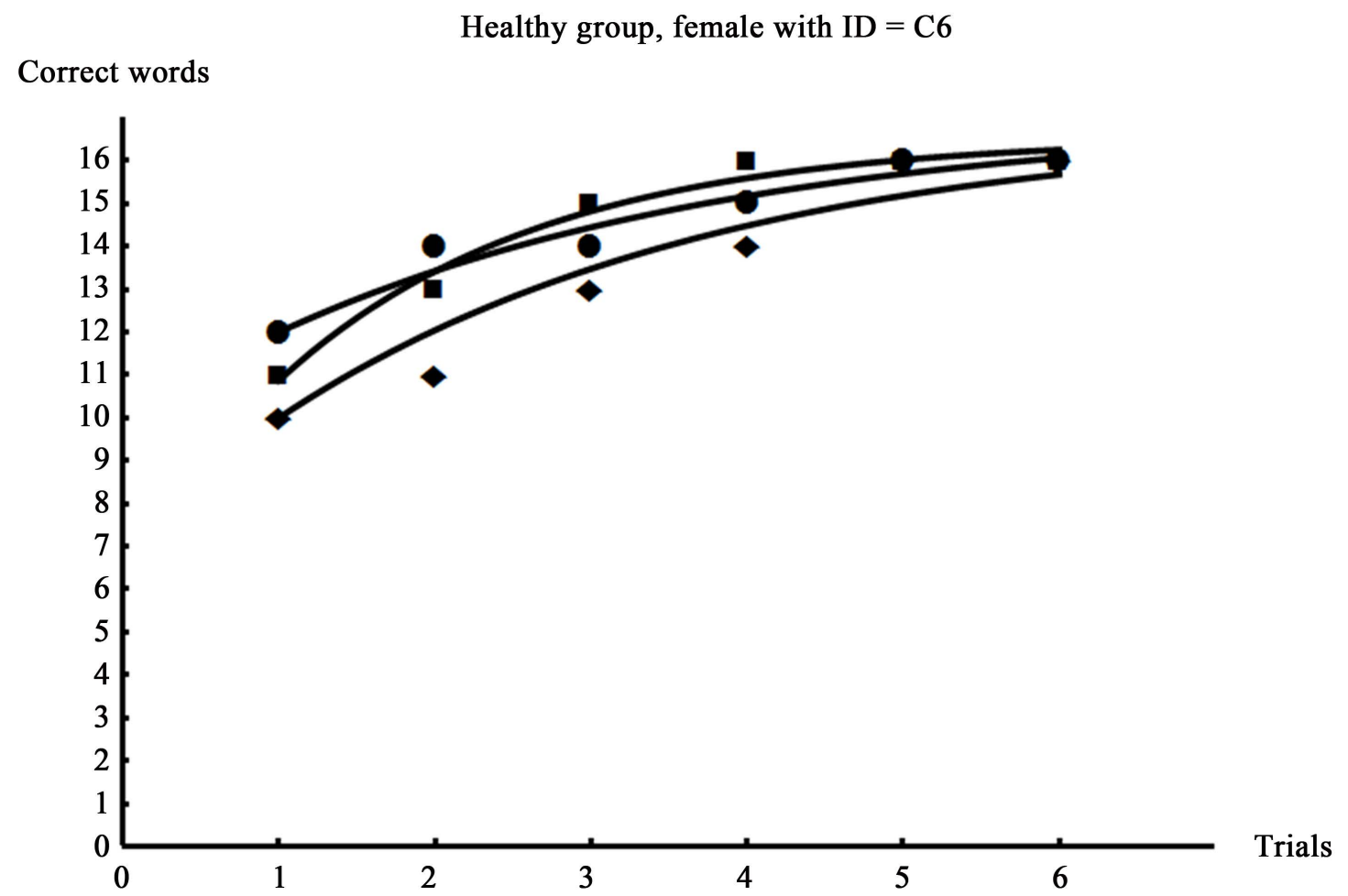

Figure 3. Healthy group, female with ID $=$ C6. Squares-the first test, diamonds-the second test, circles-the third test.

Healthy group, female with ID $=\mathrm{C} 8$

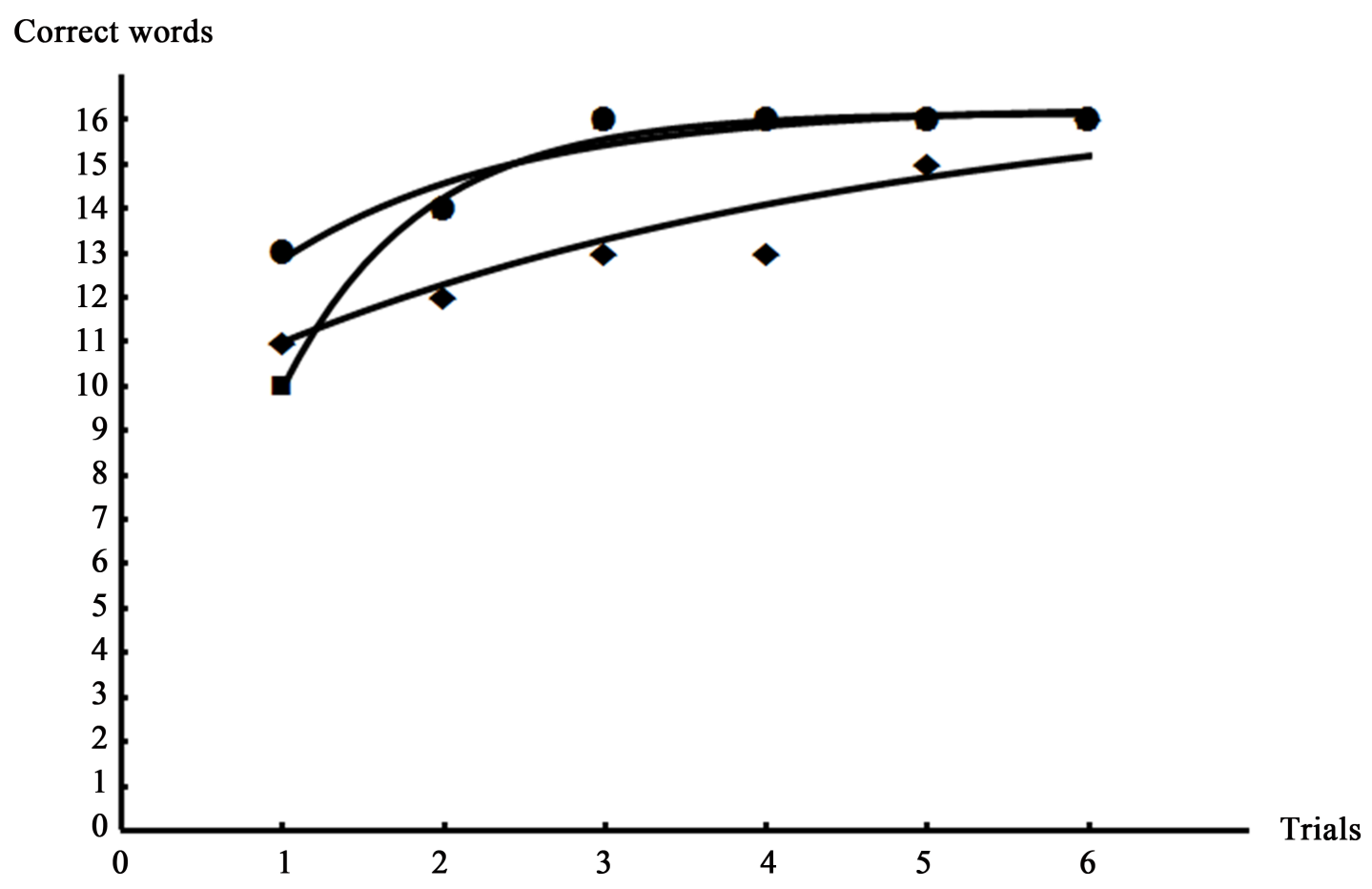

Figure 4. Healthy group, female with ID = C8. Squares-the first test, diamonds-the second test, circles-the third test. 


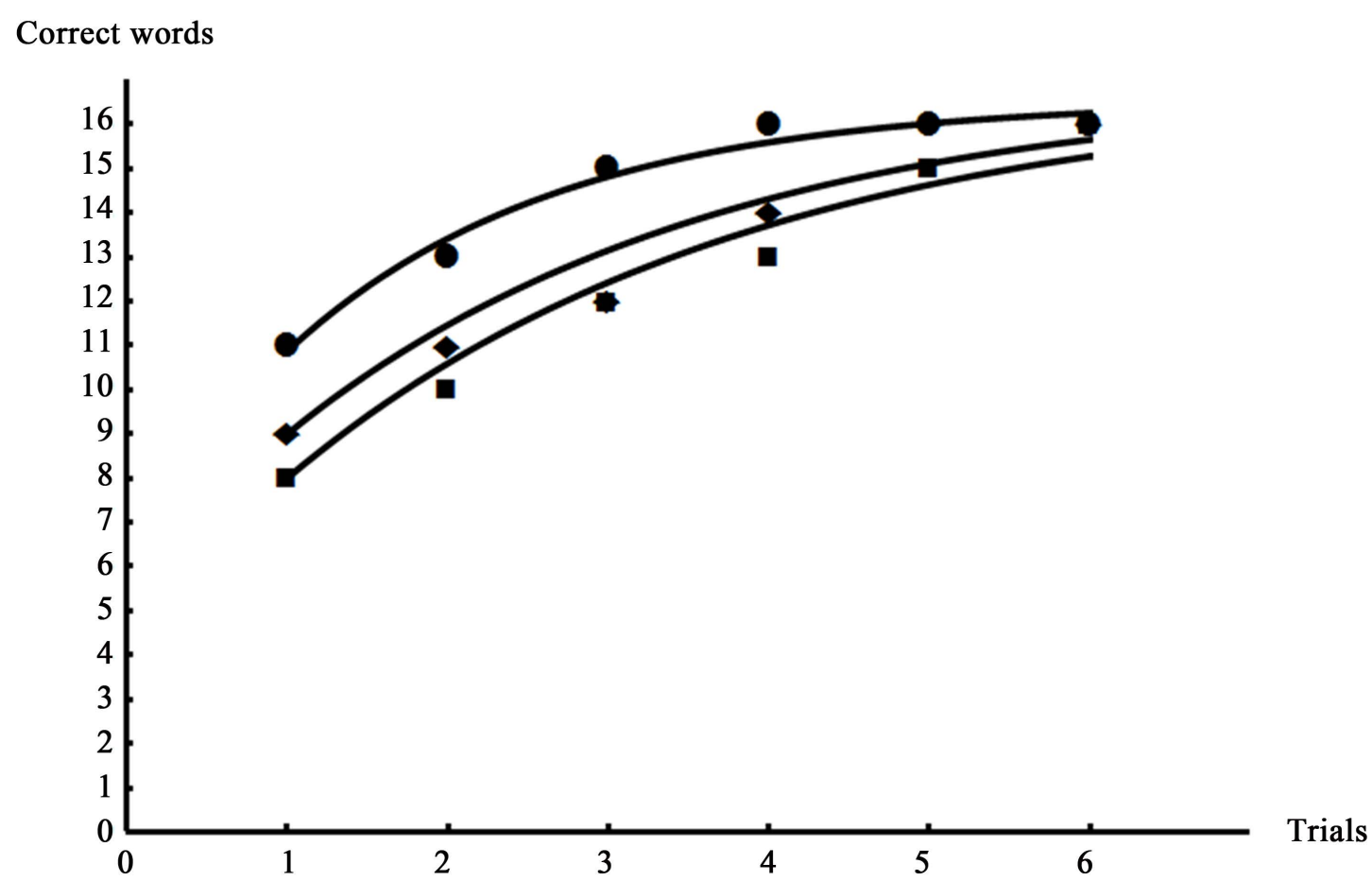

Figure 5. Healthy group, female with ID $=\mathrm{C} 11$. Squares - the first test, diamonds-the second test, circles-the third test.

Healthy group, female with ID $=\mathrm{C} 12$

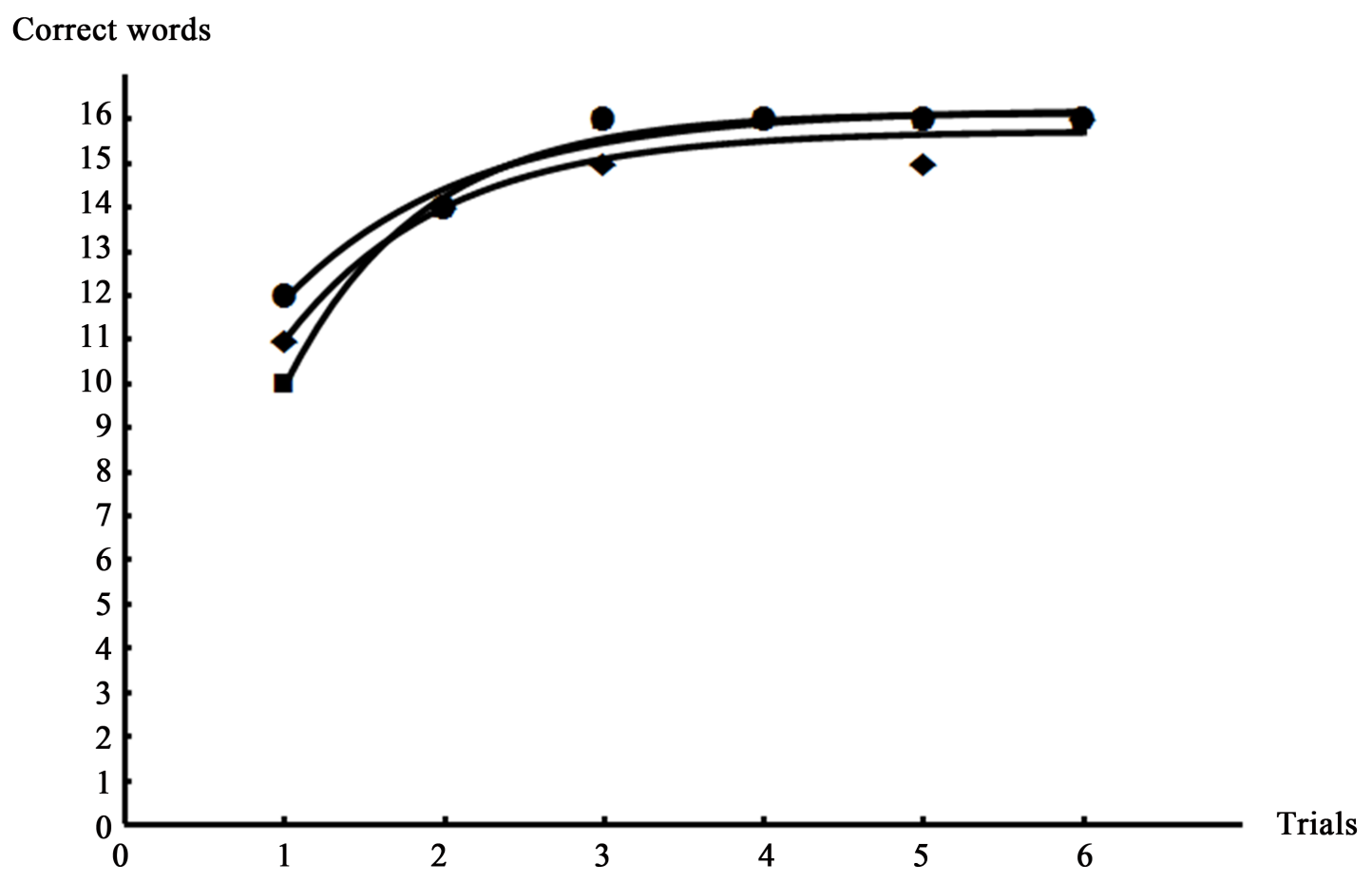

Figure 6. Healthy group, female with ID = C12. Squares-the first test, diamonds-the second test, circles-the third test. 


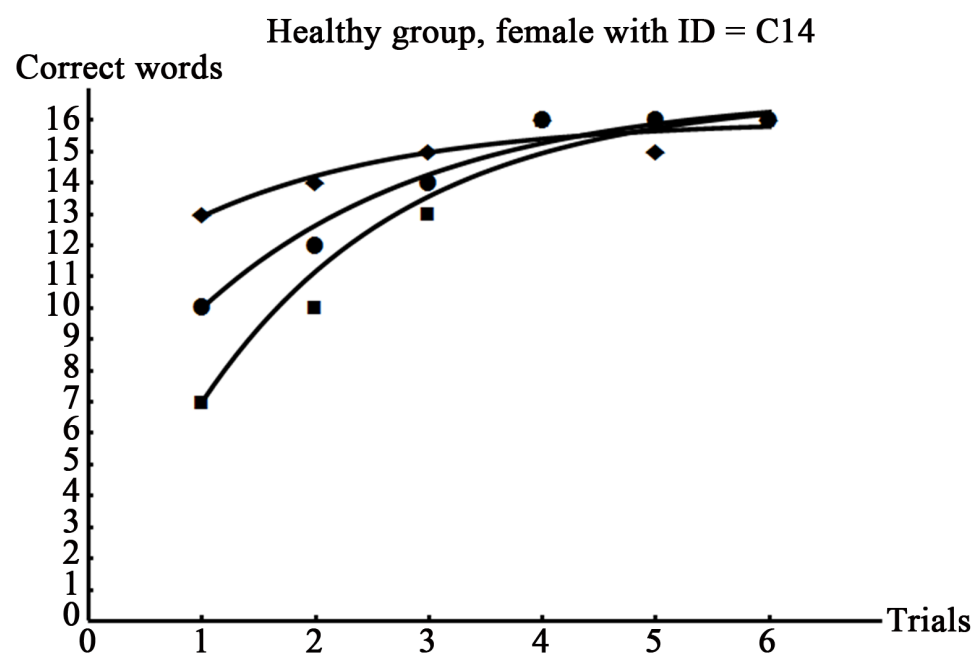

Figure 7. Healthy group, female with ID $=\mathrm{C} 14$. Squares-the first test, diamonds-the second test, circles-the third test.

Table 3. Levels of short-term memory in healthy participants, females.

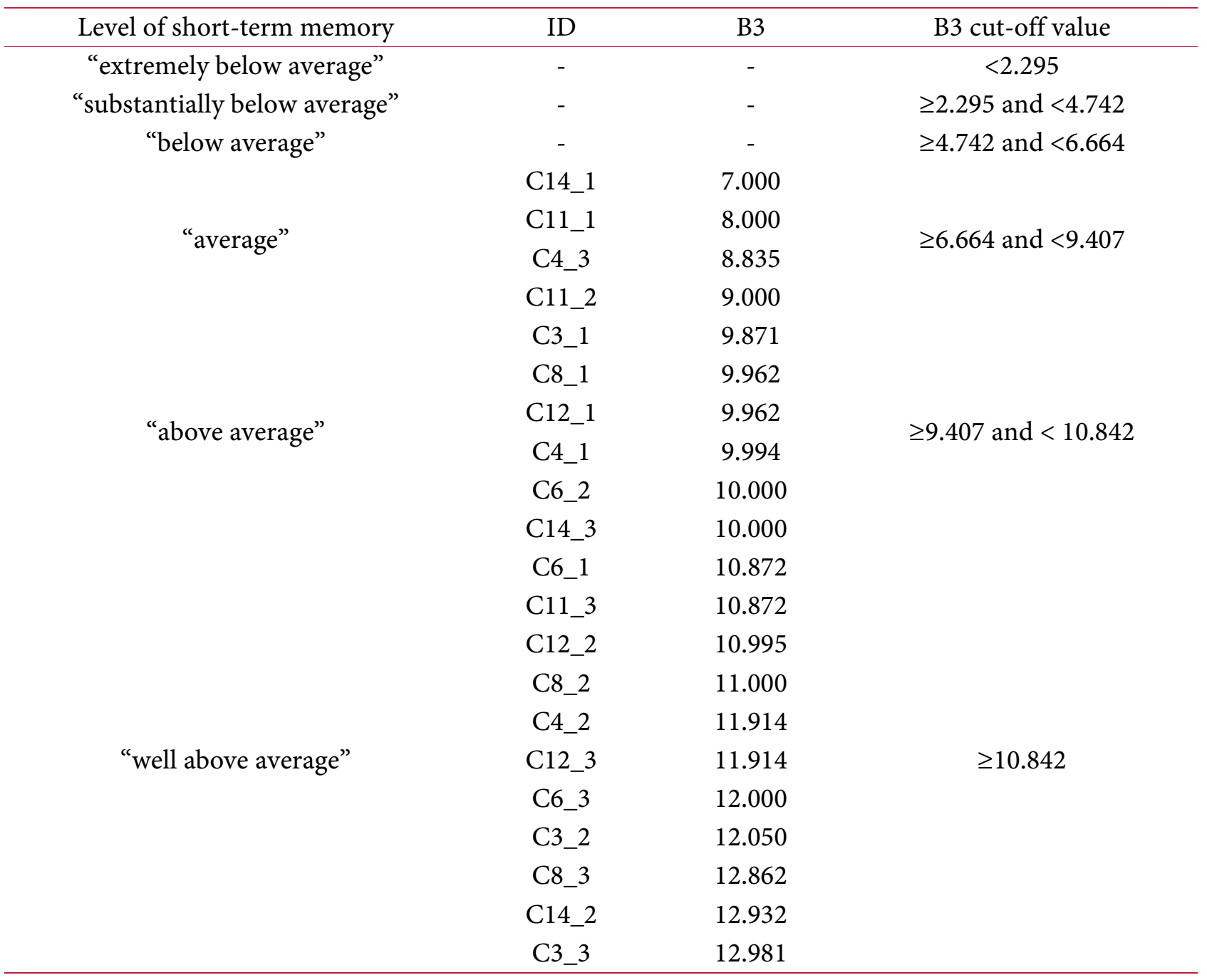


Table 4. Levels of long-term memory in healthy participants, females.

\begin{tabular}{|c|c|c|c|}
\hline Level of long-term memory & ID & B4 & B4 cut-off value \\
\hline "extremely below average" & - & - & $<5.692$ \\
\hline "substantially below average" & - & - & $\geq 5.692$ and $<6.124$ \\
\hline "below average" & - & - & $\geq 6.124$ and $<12.235$ \\
\hline “average" & - & - & $\geq 12.235$ and $<13.939$ \\
\hline "above average" & & & $\geq 13.939$ and $<15.1$ \\
\hline \multirow{21}{*}{ "well above average" } & C12_2 & 15.742 & \multirow{21}{*}{$\geq 15.1$} \\
\hline & $\mathrm{C} 14 \_2$ & 16.002 & \\
\hline & C3_3 & 16.078 & \\
\hline & C8_1 & 16.155 & \\
\hline & C12_1 & 16.155 & \\
\hline & C3_2 & 16.177 & \\
\hline & C4_2 & 16.227 & \\
\hline & C12_3 & 16.227 & \\
\hline & C4_3 & 16.283 & \\
\hline & C8_3 & 16.299 & \\
\hline & C3_1 & 16.340 & \\
\hline & C6_1 & 16.544 & \\
\hline & C11_3 & 16.544 & \\
\hline & C4_1 & 16.602 & \\
\hline & C11_1 & 16.821 & \\
\hline & C14_1 & 16.823 & \\
\hline & C6_2 & 16.835 & \\
\hline & C11_2 & 16.852 & \\
\hline & C14_3 & 16.868 & \\
\hline & C8_2 & 16.868 & \\
\hline & C6_3 & 16.996 & \\
\hline
\end{tabular}

Table 3 shows that four tests were assigned to the "average" level, six tests were assigned to the "above average" level, and 11 tests were assigned to the "well above average" level. The value of Trial 1 was in the range of 11 - 13 words in 11 tests. This result proves that the size of a list of ten words is not sufficient for an accurate assessment of short-term memory.

Table 4 shows that all B4 values were assigned to the "well above average" level. This means that each participant recalled all words from the list.

Healthy participants, males 
Raw learning data are provided in Table 5, and our model's coefficients for individual learning curves are given in Table 6.

Figures below show three individual learning curves for each participant and the corresponding averaged curve (see Figures 8-15).

Table 5. Raw learning data for healthy group, males.

\begin{tabular}{cccccccc}
\hline ID & Test/retest & Trial 1 & Trial 2 & Trial 3 & Trial 4 & Trial 5 & Trial 6 \\
\hline & 1 & 11 & 12 & 14 & 16 & 14 & 16 \\
C1 & 2 & 13 & 16 & 16 & 16 & 16 & 16 \\
& 3 & 14 & 16 & 16 & 16 & 16 & 16
\end{tabular}

Mean \pm S.E.M. $12.67 \pm 0.88214 .67 \pm 1.33315 .33 \pm 0.667 \quad 16.00 \pm 0.00015 .33 \pm 0.667 \quad 16.00 \pm 0.000$

$\begin{array}{rlllllll} & 1 & 10 & 13 & 15 & 16 & 16 & 16 \\ \mathrm{C} 2 & 2 & 11 & 13 & 15 & 15 & 16 & 16 \\ & 3 & 12 & 15 & 16 & 16 & 16 & 16\end{array}$

Mean \pm S.E.M. $11.00 \pm 0.57713 .67 \pm 0.667 \quad 15.33 \pm 0.33315 .67 \pm 0.33316 .00 \pm 0.00016 .00 \pm 0.000$

$\begin{array}{rrrrrrrr} & 1 & 8 & 12 & 13 & 14 & 15 & 15 \\ \mathrm{C} 5 & 2 & 10 & 12 & 14 & 16 & 16 & 16 \\ & 3 & 12 & 14 & 14 & 15 & 16 & 16\end{array}$

Mean \pm S.E.M. $10.00 \pm 1.15512 .67 \pm 0.667 \quad 13.67 \pm 0.333 \quad 15.00 \pm 0.577 \quad 15.67 \pm 0.333 \quad 15.67 \pm 0.333$

$\begin{array}{rrrrrrrr} & 1 & 10 & 12 & 14 & 16 & 16 & 16 \\ \mathrm{C} 7 & 2 & 13 & 14 & 16 & 16 & 16 & 16 \\ & 3 & 12 & 15 & 16 & 16 & 16 & 16\end{array}$

Mean \pm S.E.M. $11.67 \pm 0.88213 .67 \pm 0.88215 .33 \pm 0.667 \quad 16.00 \pm 0.00016 .00 \pm 0.00016 .00 \pm 0.000$

C9

$\begin{array}{rrrrrrr}1 & 12 & 14 & 16 & 16 & 16 & 16 \\ 2 & 10 & 13 & 15 & 16 & 16 & 16 \\ 3 & 13 & 15 & 16 & 16 & 16 & 16\end{array}$

Mean \pm S.E.M. $11.67 \pm 0.88214 .00 \pm 0.577 \quad 15.67 \pm 0.33316 .00 \pm 0.00016 .00 \pm 0.00016 .00 \pm 0.000$

$\mathrm{C} 10$

$\begin{array}{rrrrrrr}1 & 11 & 14 & 15 & 14 & 16 & 16 \\ 2 & 13 & 15 & 16 & 16 & 16 & 16 \\ 3 & 14 & 15 & 16 & 16 & 16 & 16\end{array}$

Mean \pm S.E.M. $12.67 \pm 0.88214 .67 \pm 0.33315 .67 \pm 0.33315 .33 \pm 0.667 \quad 16.00 \pm 0.00016 .00 \pm 0.000$

$\begin{array}{rccccccc} & 1 & 9 & 13 & 15 & 16 & 16 & 16 \\ \mathrm{C} 13 & 2 & 10 & 12 & 15 & 16 & 16 & 16 \\ & 3 & 8 & 11 & 13 & 14 & 16 & 16\end{array}$

Mean \pm S.E.M. $\quad 9.00 \pm 0.577 \quad 12.00 \pm 0.577 \quad 14.33 \pm 0.667 \quad 15.33 \pm 0.667 \quad 16.00 \pm 0.00016 .00 \pm 0.000$

$\begin{array}{rlllllll} & 1 & 11 & 12 & 14 & 16 & 16 & 16 \\ \mathrm{C} 15 & 2 & 12 & 15 & 16 & 16 & 16 & 16 \\ & 3 & 10 & 12 & 13 & 16 & 16 & 16\end{array}$

Mean \pm S.E.M. $11.00 \pm 0.577 \quad 13.00 \pm 1.00014 .33 \pm 0.882 \quad 16.00 \pm 0.000 \quad 16.00 \pm 0.00016 .00 \pm 0.000$ 
Table 6. Individual learning curves for healthy group, males.

\begin{tabular}{|c|c|c|c|c|c|}
\hline \multirow{2}{*}{ ID } & \multirow{2}{*}{ Test/retest } & \multicolumn{3}{|c|}{ Model's coefficients } & \multirow{2}{*}{$\mathrm{R}^{2}$} \\
\hline & & B2 & B3 & B4 & \\
\hline \multirow{4}{*}{$\mathrm{C} 1$} & 1 & $0.402 \pm 0.437$ & $10.783 \pm 1.095$ & $16.414 \pm 2.668$ & 0.814 \\
\hline & 2 & $0.685 \pm 0.547$ & $13.000 \pm 0.796$ & $16.572 \pm 0.863$ & 0.739 \\
\hline & 3 & $0.429 \pm 0.556$ & $14.000 \pm 0.685$ & $16.760 \pm 1.496$ & 0.548 \\
\hline & Averaged & $1.010 \pm 0.342$ & $12.667 \pm 0.337$ & $15.826 \pm 0.248$ & 0.956 \\
\hline \multirow{4}{*}{$\mathrm{C} 2$} & 1 & $0.729 \pm 0.113$ & $9.926 \pm 0.288$ & $16.387 \pm 0.290$ & 0.991 \\
\hline & 2 & $0.542 \pm 0.149$ & $10.949 \pm 0.360$ & $16.433 \pm 0.539$ & 0.979 \\
\hline & 3 & $1.425 \pm 0.141$ & $11.991 \pm 0.119$ & $16.059 \pm 0.072$ & 0.997 \\
\hline & Averaged & $0.799 \pm 0.083$ & $10.966 \pm 0.161$ & $16.178 \pm 0.147$ & 0.996 \\
\hline \multirow{4}{*}{ C5 } & 1 & $0.674 \pm 0.132$ & $8.103 \pm 0.385$ & $15.206 \pm 0.426$ & 0.987 \\
\hline & 2 & $0.486 \pm 0.193$ & $10.000 \pm 0.590$ & $16.868 \pm 1.045$ & 0.966 \\
\hline & 3 & $0.337 \pm 0.207$ & $12.000 \pm 0.448$ & $16.996 \pm 1.498$ & 0.943 \\
\hline & Averaged & $0.491 \pm 0.090$ & $10.034 \pm 0.255$ & $16.350 \pm 0.444$ & 0.992 \\
\hline \multirow{4}{*}{ C7 } & 1 & $0.486 \pm 0.193$ & $10.000 \pm 0.590$ & $16.868 \pm 1.045$ & 0.966 \\
\hline & 2 & $0.689 \pm 0.348$ & $12.862 \pm 0.486$ & $16.299 \pm 0.524$ & 0.917 \\
\hline & 3 & $1.425 \pm 0.141$ & $11.991 \pm 0.119$ & $16.059 \pm 0.072$ & 0.997 \\
\hline & Averaged & $0.696 \pm 0.142$ & $11.591 \pm 0.272$ & $16.330 \pm 0.289$ & 0.985 \\
\hline \multirow{4}{*}{ C9 } & 1 & $0.875 \pm 0.270$ & $11.914 \pm 0.409$ & $16.227 \pm 0.340$ & 0.964 \\
\hline & 2 & $0.729 \pm 0.113$ & $9.926 \pm 0.288$ & $16.387 \pm 0.290$ & 0.991 \\
\hline & 3 & $1.180 \pm 0.185$ & $12.981 \pm 0.152$ & $16.078 \pm 0.101$ & 0.990 \\
\hline & Averaged & $0.863 \pm 0.156$ & $11.613 \pm 0.255$ & $16.207 \pm 0.214$ & 0.987 \\
\hline \multirow{4}{*}{$\mathrm{C} 10$} & 1 & $0.807 \pm 0.498$ & $11.106 \pm 0.853$ & $15.707 \pm 0.767$ & 0.872 \\
\hline & 2 & $1.180 \pm 0.185$ & $12.981 \pm 0.152$ & $16.078 \pm 0.101$ & 0.990 \\
\hline & 3 & $0.875 \pm 0.270$ & $13.957 \pm 0.205$ & $16.113 \pm 0.170$ & 0.964 \\
\hline & Averaged & $0.963 \pm 0.271$ & $12.674 \pm 0.287$ & $15.932 \pm 0.219$ & 0.969 \\
\hline \multirow{4}{*}{$\mathrm{C} 13$} & 1 & $0.851 \pm 0.083$ & $8.960 \pm 0.218$ & $16.283 \pm 0.186$ & 0.996 \\
\hline & 2 & $0.568 \pm 0.212$ & $9.764 \pm 0.647$ & $16.774 \pm 0.905$ & 0.960 \\
\hline & 3 & $0.451 \pm 0.127$ & $8.000 \pm 0.501$ & $16.820 \pm 1.004$ & 0.983 \\
\hline & Averaged & $0.566 \pm 0.070$ & $8.920 \pm 0.235$ & $16.662 \pm 0.331$ & 0.995 \\
\hline \multirow{4}{*}{$\mathrm{C} 15$} & 1 & $0.432 \pm 0.294$ & $11.000 \pm 0.767$ & $16.830 \pm 1.652$ & 0.924 \\
\hline & 2 & $1.425 \pm 0.141$ & $11.991 \pm 0.119$ & $16.059 \pm 0.072$ & 0.997 \\
\hline & 3 & $0.451 \pm 0.279$ & $10.000 \pm 0.853$ & $16.846 \pm 1.712$ & 0.929 \\
\hline & Averaged & $0.491 \pm 0.152$ & $10.907 \pm 0.399$ & $16.779 \pm 0.697$ & 0.976 \\
\hline
\end{tabular}




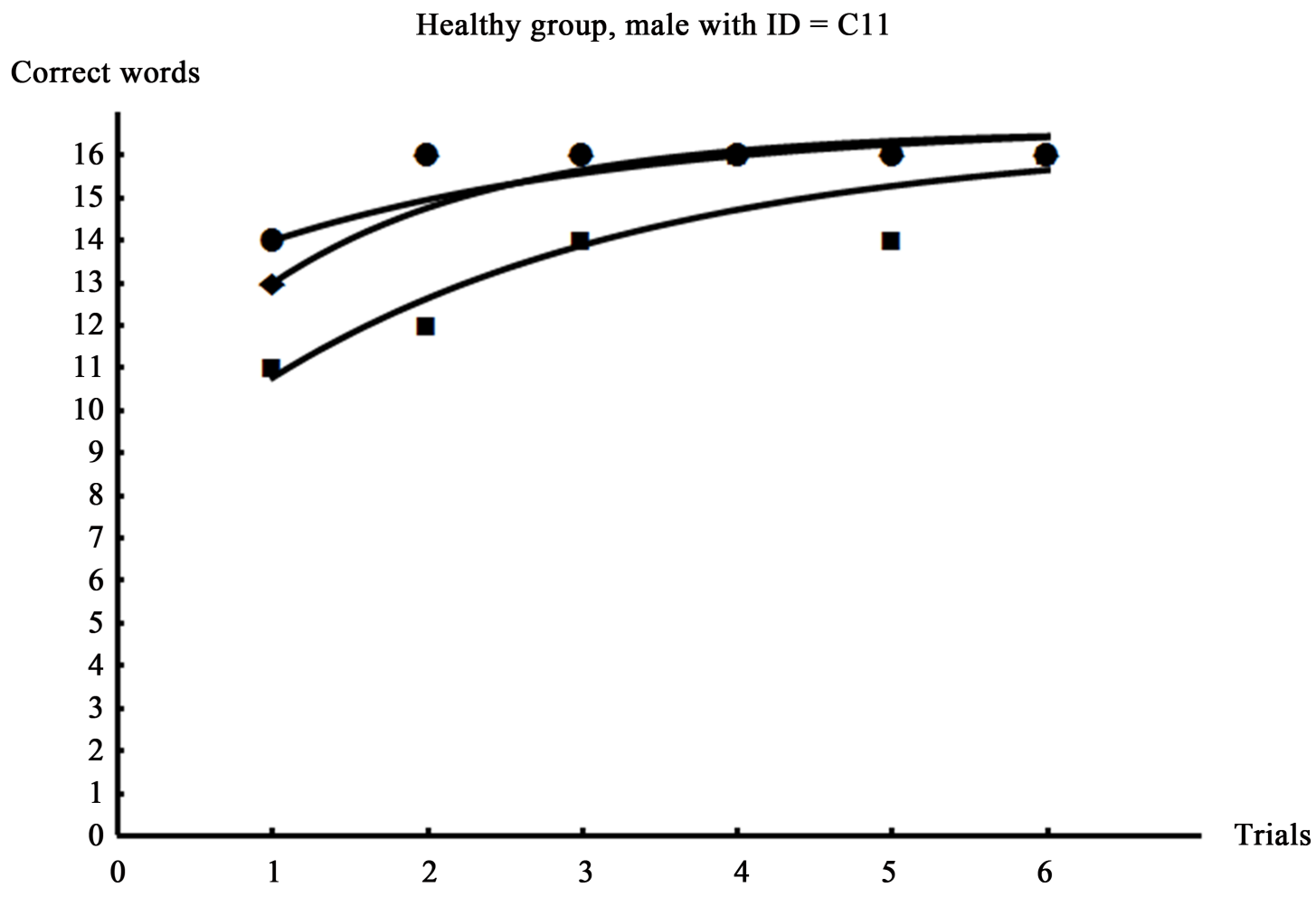

Figure 8. Healthy group, male with ID $=\mathrm{C} 1$. Squares-the first test, diamonds-the second test, circles-the third test.

Healthy group, male with ID $=\mathrm{C} 2$

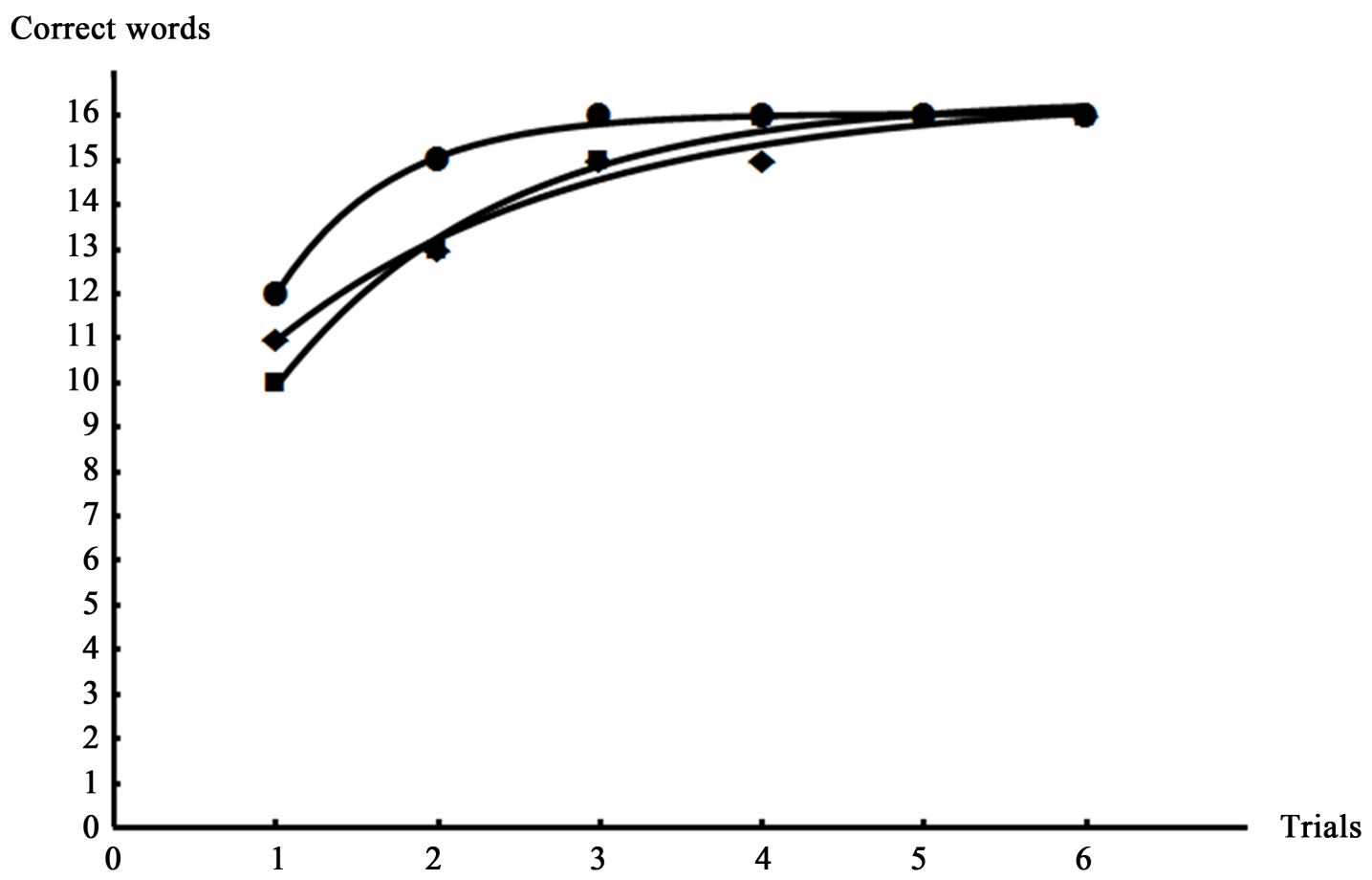

Figure 9. Healthy group, male with ID $=\mathrm{C} 2$. Squares-the first test, diamonds-the second test, circles-the third test. 


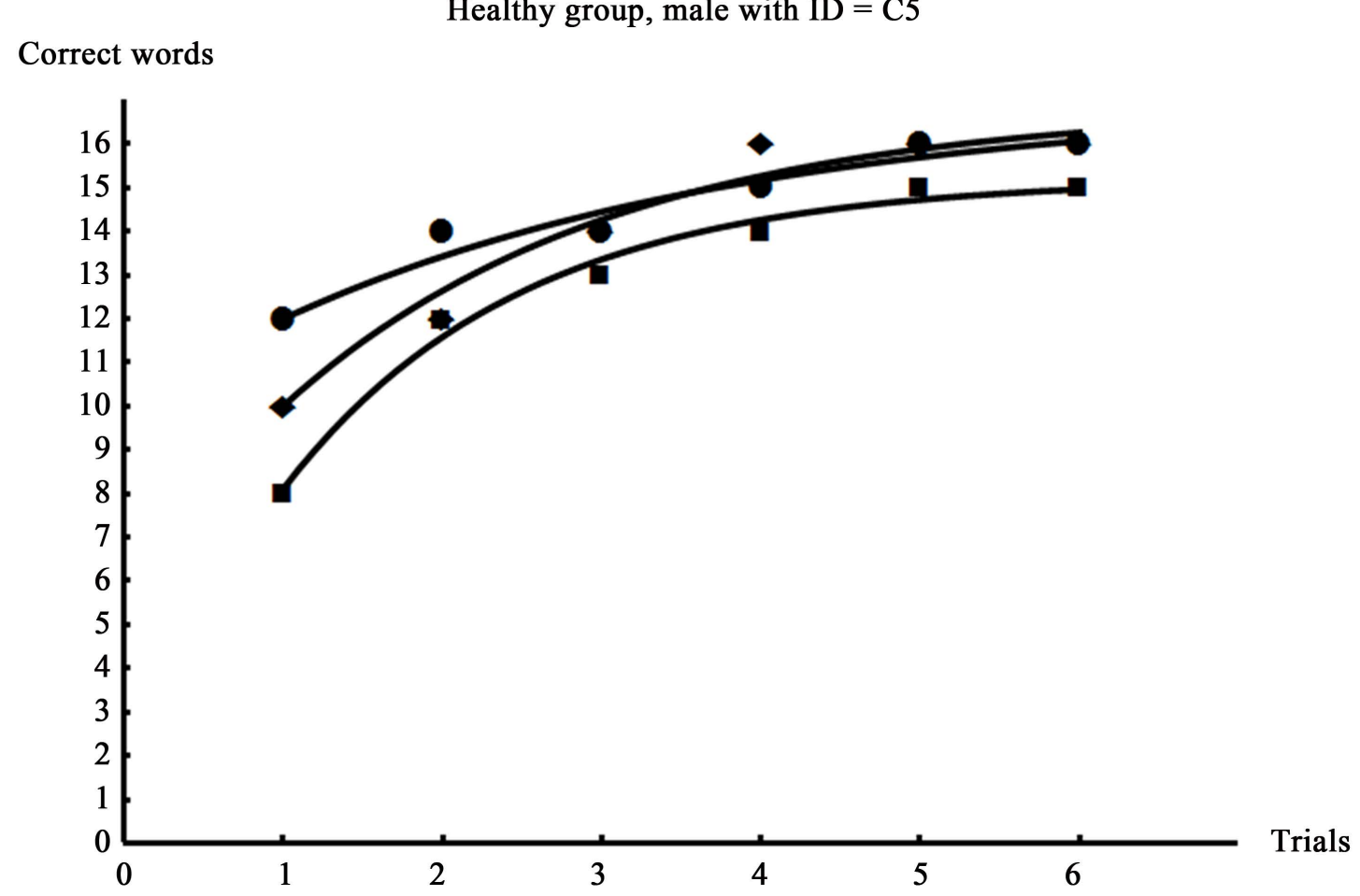

Figure 10. Healthy group, male with ID = C5. Squares-the first test, diamonds-the second test, circles-the third test.

Healthy group, male with ID $=\mathrm{C} 7$

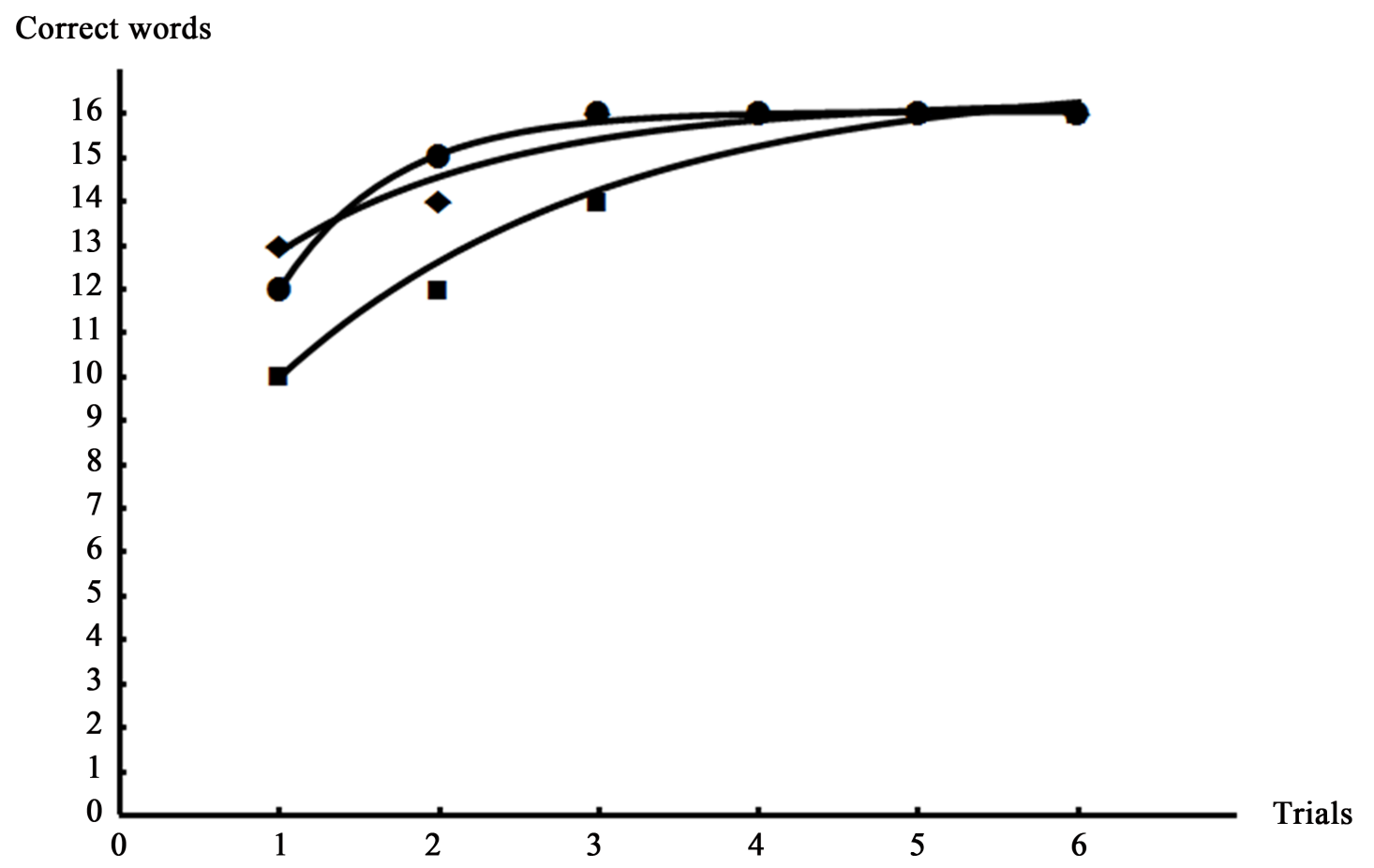

Figure 11. Healthy group, male with ID = C7. Squares-the first test, diamonds-the second test, circles-the third test. 
Healthy group, male with ID $=\mathrm{C} 9$

Correct words

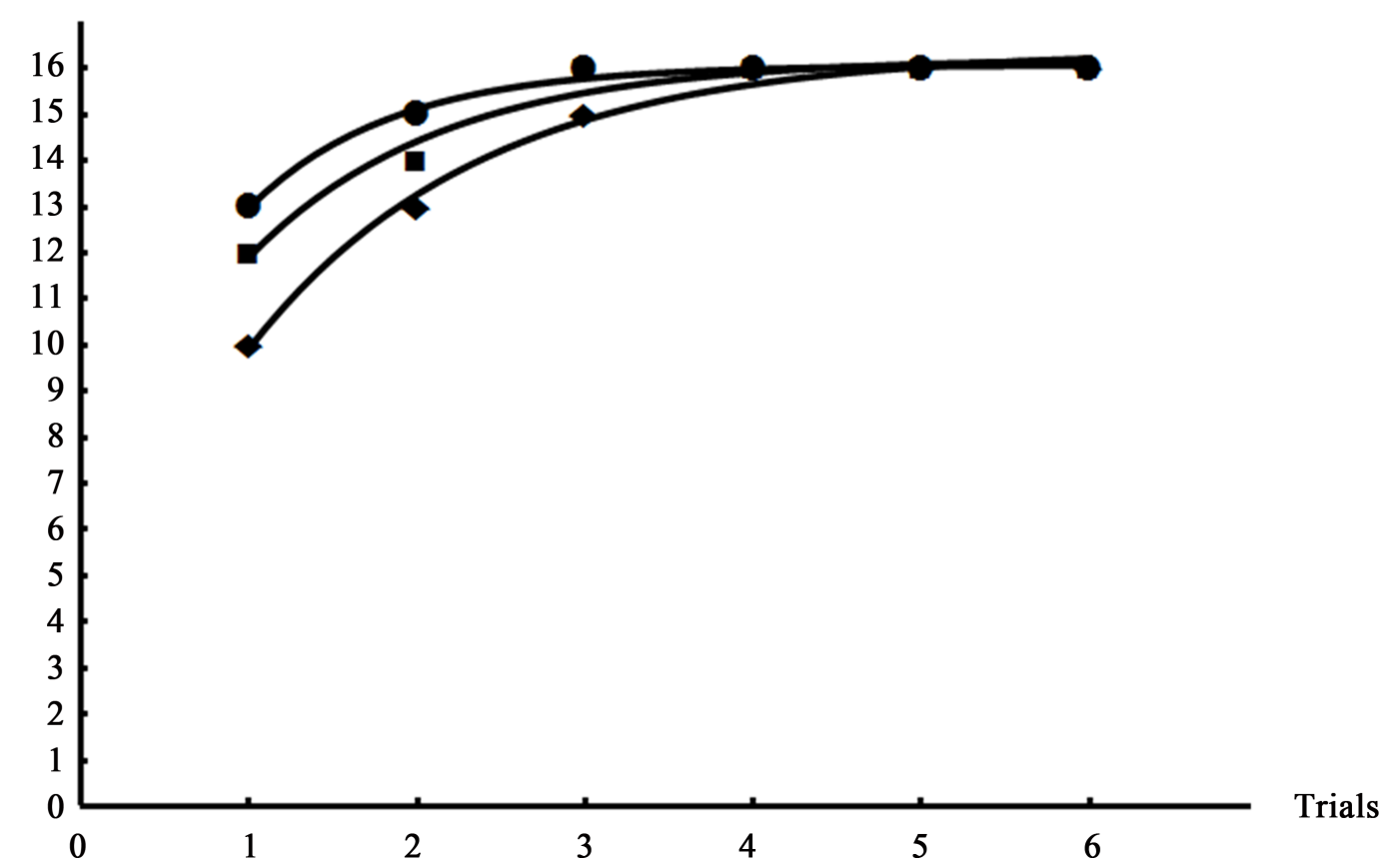

Figure 12. Healthy group, male with ID = C9. Squares-the first test, diamonds-the second test, circles-the third test.

$$
\text { Healthy group, male with ID }=\mathrm{C} 10
$$

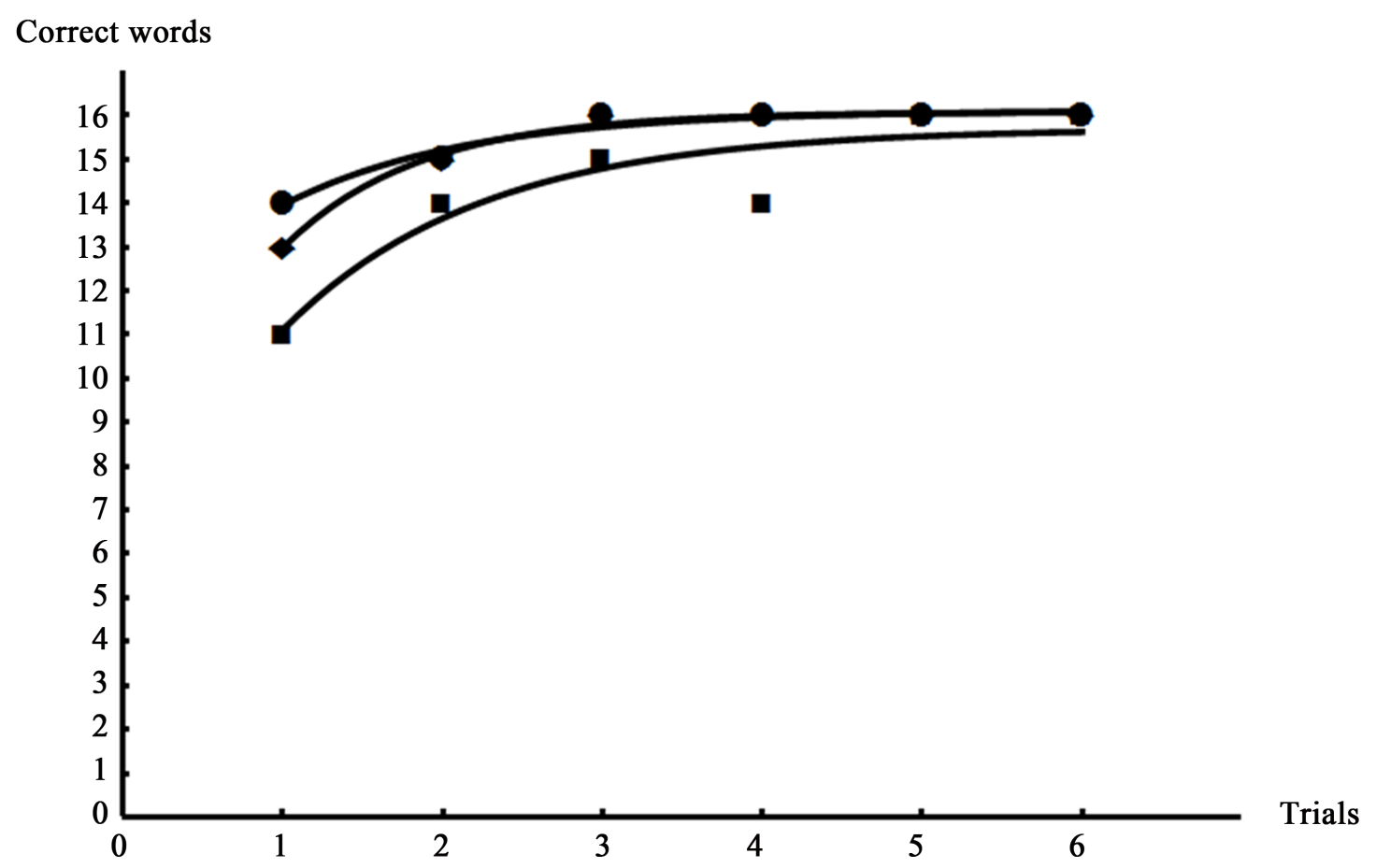

Figure 13. Healthy group, male with ID = C10. Squares—the first test, diamonds-the second test, circles-the third test. 


\section{Correct words}

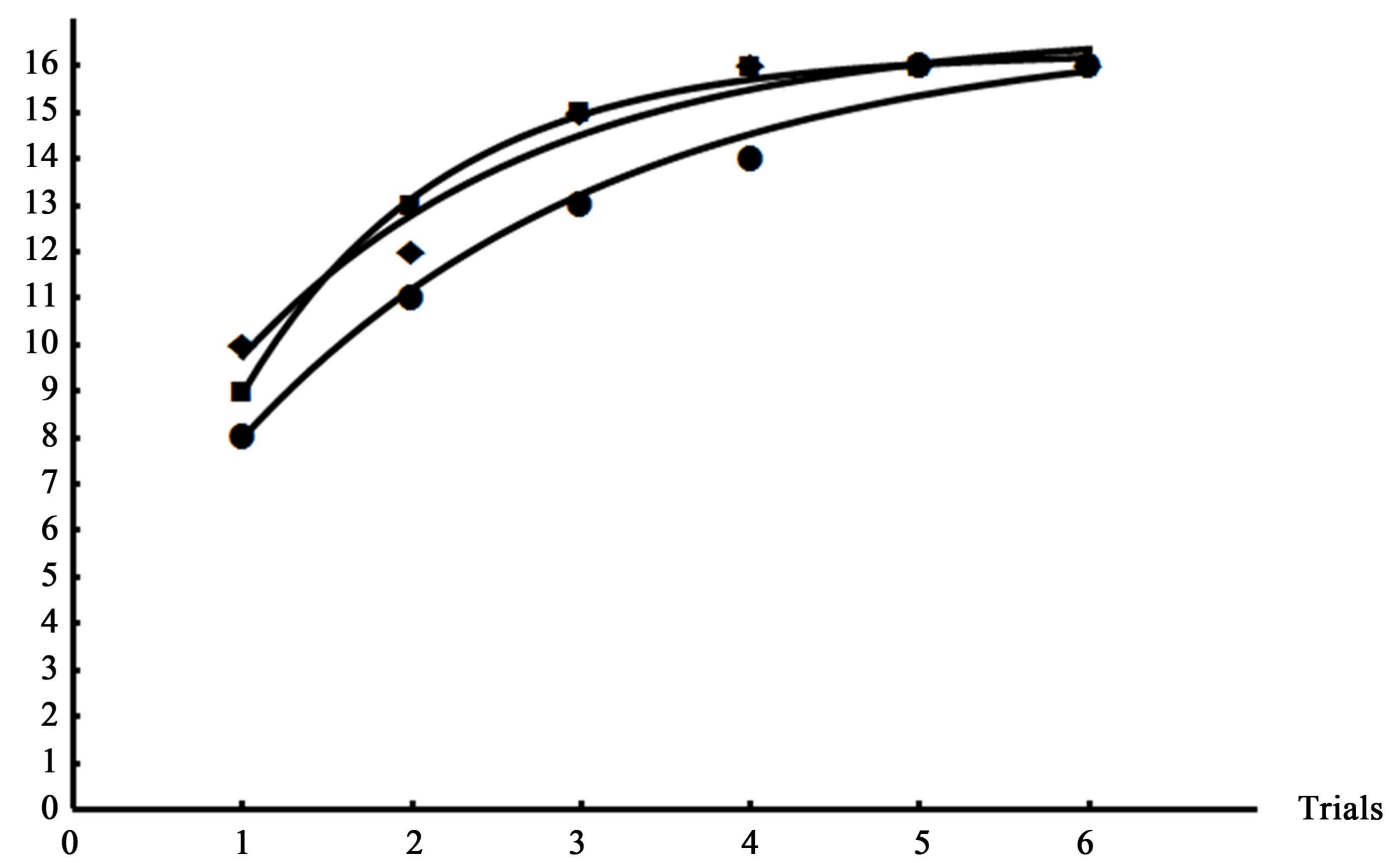

Figure 14. Healthy group, male with ID $=$ C13. Squares-the first test, diamonds-the second test, circles-the third test.

$$
\text { Healthy group, male with ID }=\mathrm{C} 15
$$

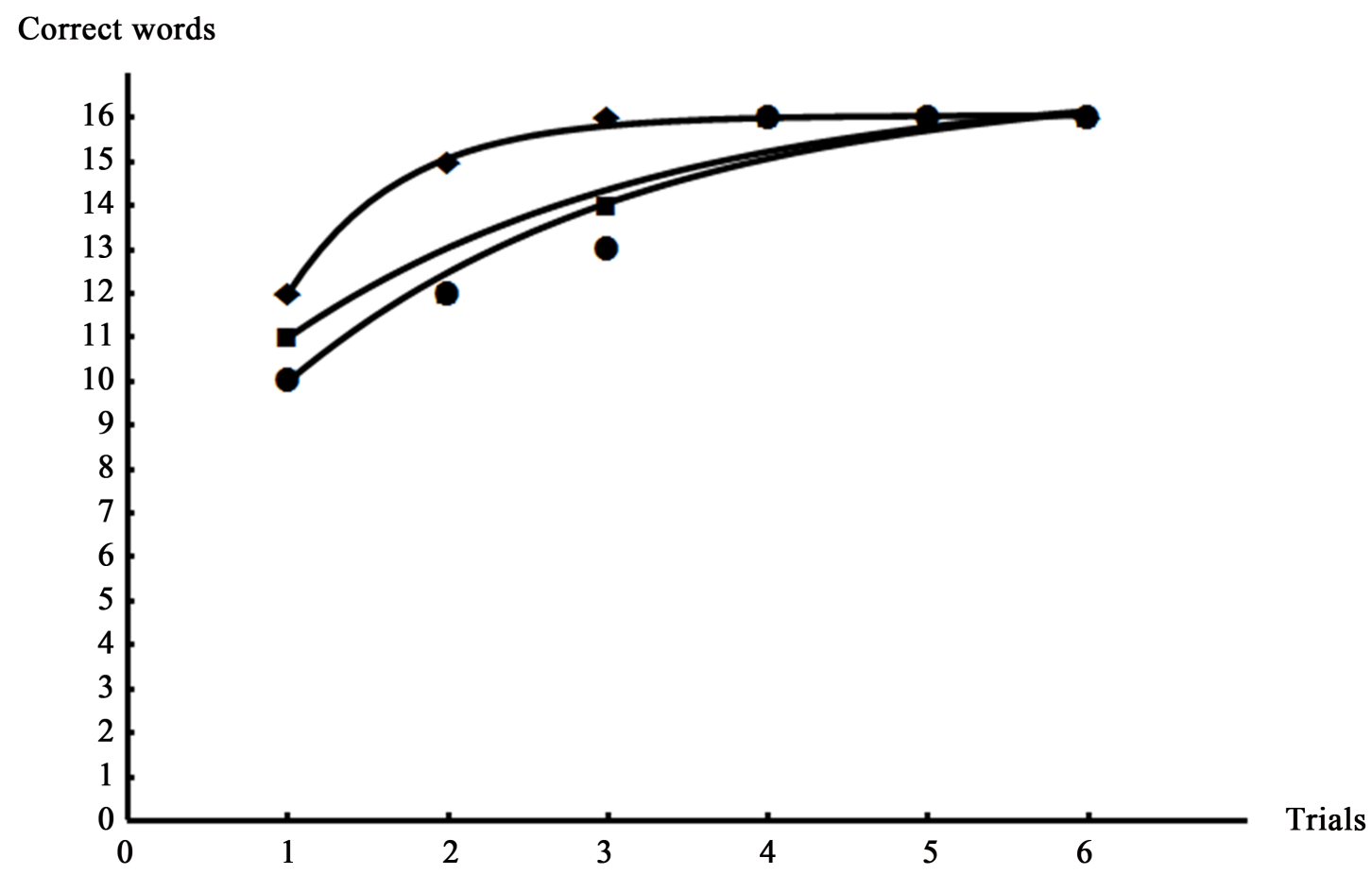

Figure 15. Healthy group, male with ID $=$ C15. Squares-the first test, diamonds-the second test, circles-the third test. 
During the three tests, each participant recalled a different number of words on Trial 1, but recalled practically all the words from the list on the later trials. Four participants (ID = C1, C2, C5, C10) monotonously increased Trial 1 values from test 1 to test 3 . This order was different in other participants (ID = C7, C9, C13, C15). Thus, in healthy males, averaging over three tests also results in more precise short-term memory assessment (coefficient B3).

Analysis of short-term memory levels is presented in Table 7 and long-term memory levels in Table 8.

Table 7. Levels of short-term memory in healthy participants, males.

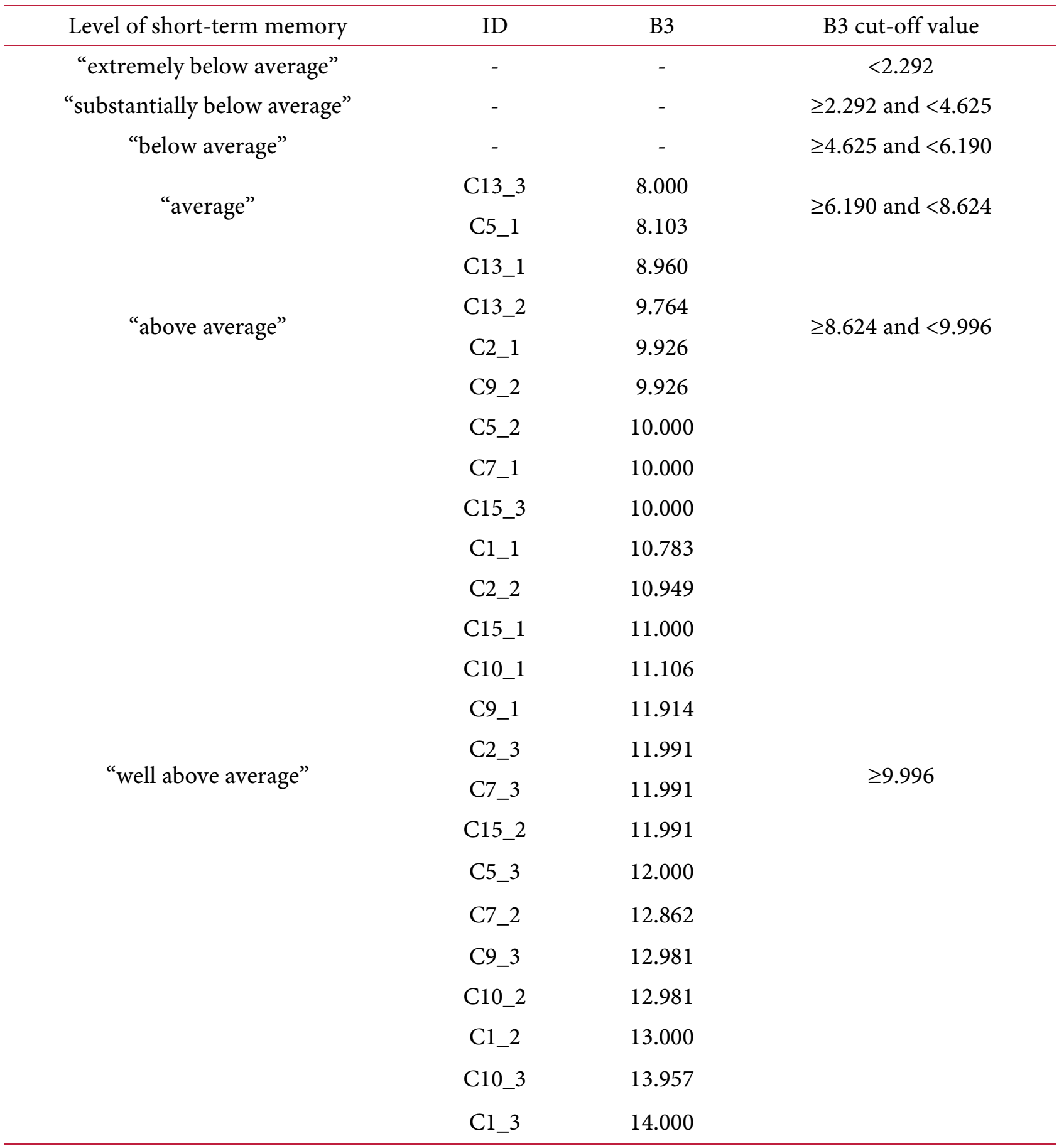


Table 8. Levels of long-term memory in healthy participants, males.

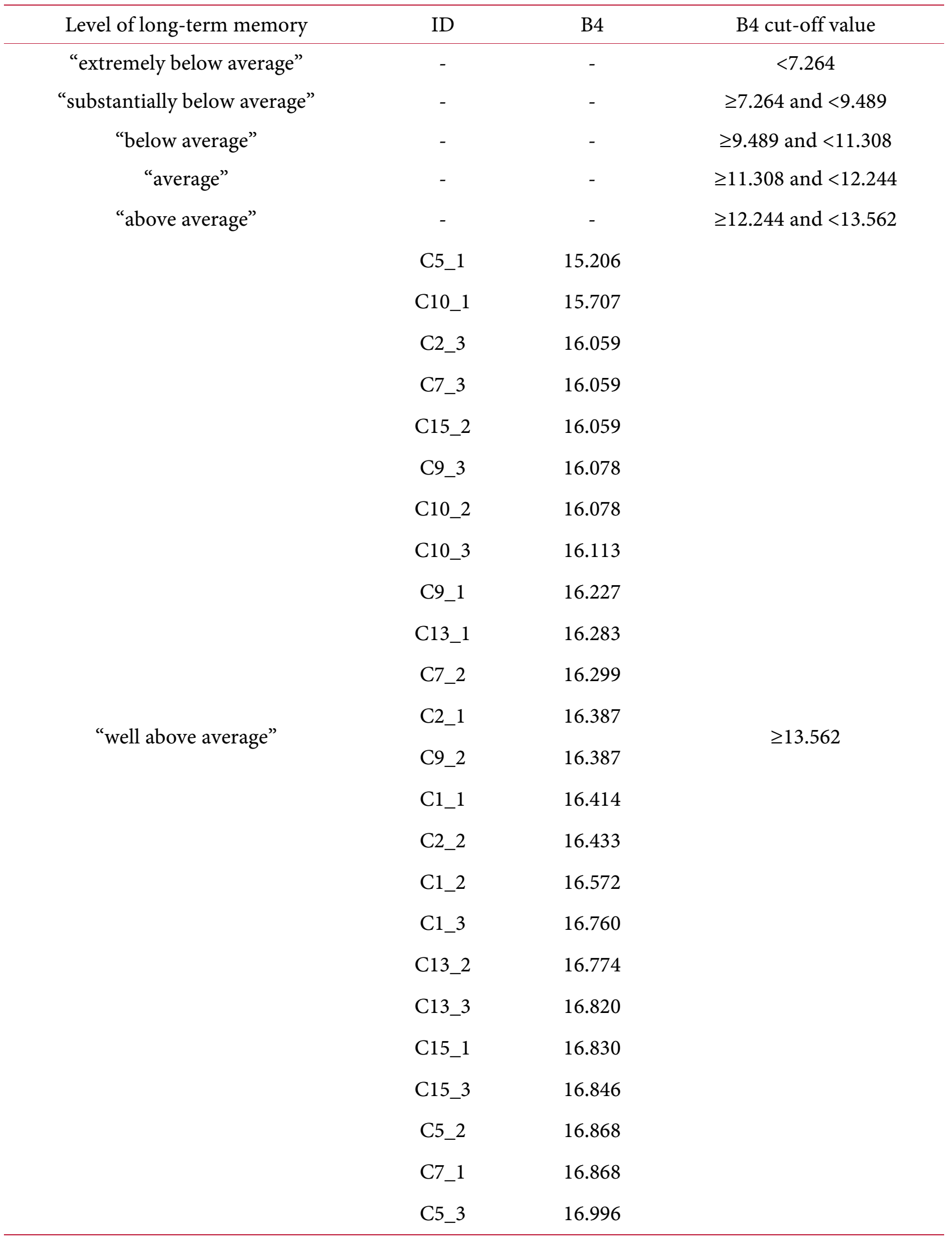


Table 7 shows that two tests were assigned to the "average" level, five tests were assigned to the "above average" level, and the remainder to the "well above average" level. Note that in 13 tests the B3 value exceeded 10.0. This result confirms that a list of ten words is not sufficient for a correct assessment of short-term memory.

Table 8 reveals that all B4 values were assigned to the "well above average" level. B4 values were greater than 16.0 in 22 tests. The B4 value is less than 16.0 in only two tests. It is important to note that in Trial 6, 15 words were recalled in only one test (C5_1), and 16 words were recalled in all other tests.

\section{Drug addicted females}

Raw learning data are provided in Table 9 and the model's coefficients for individual learning curves are provided in Table 10.

Figures below show three individual learning curves for each participant and the corresponding averaged curve (see Figures 16-19).

Analysis of short-term memory levels is presented in Table 11 and long-term memory levels in Table 12.

Table 11 shows that three tests were assigned to the "below average" level-patient D7, four tests were assigned to the "average" level, three tests were assigned to the "above average" level, and two tests were assigned to the "well above average" level. The value of Trial 1 was in the range of 5 - 11 words. This result shows that short-term memory is impaired in patient D7. There is also instability in repeated tests/retests. For example, patient D6 was assigned to "average" and "above average" levels. Patient D4 was assigned to "average" and "well above average" levels. Patient D13 was assigned to "average" and "above average" levels. We suggest that this instability might be due to an incomplete effort on test performance.

Table 9. Raw learning data for drug addicts, females.

\begin{tabular}{cccccccc}
\hline ID & Test/retest & Trial 1 & Trial 2 & Trial 3 & Trial 4 & Trial 5 & Trial 6 \\
\hline \multirow{3}{*}{ D4 } & 1 & 11 & 13 & 14 & 16 & 12 & 16 \\
& 2 & 8 & 12 & 14 & 16 & 15 & 16 \\
& 3 & 11 & 12 & 14 & 16 & 16 & 16 \\
& Mean \pm S.E.M. & $10.00 \pm 1.000$ & $12.33 \pm 0.333$ & $14.00 \pm 0.000$ & $16.00 \pm 0.000$ & $14.33 \pm 1.20216 .00 \pm 0.000$ \\
& 1 & 7 & 12 & 14 & 16 & 16 & 16 \\
& 2 & 8 & 13 & 15 & 16 & 16 & 16
\end{tabular}

Mean \pm S.E.M. $8.33 \pm 0.88213 .00 \pm 0.57714 .67 \pm 0.33315 .67 \pm 0.33316 .00 \pm 0.00016 .00 \pm 0.000$

$\begin{array}{cccccccc} & 1 & 6 & 9 & 12 & 11 & 16 & 16 \\ \text { D7 } & 2 & 6 & 8 & 11 & 14 & 16 & 16 \\ & 3 & 5 & 8 & 9 & 14 & 14 & 15 \\ & \text { Mean } \pm \text { S.E.M. } & 5.67 \pm 0.333 & 8.33 \pm 0.333 & 10.67 \pm 0.882 & 13.00 \pm 1.000 & 15.33 \pm 0.667 & 15.67 \pm 0.333 \\ & 1 & 10 & 10 & 12 & 12 & 10 & 14 \\ \text { D13 } & 2 & 10 & 11 & 12 & 14 & 15 & 14\end{array}$

Mean \pm S.E.M. $9.67 \pm 0.33311 .00 \pm 0.57712 .00 \pm 0.00013 .00 \pm 0.57713 .33 \pm 1.66714 .67 \pm 0.667$ 
Table 10. Individual learning curves for drug addicts, females.

\begin{tabular}{|c|c|c|c|c|c|}
\hline \multirow{2}{*}{ ID } & \multirow{2}{*}{ Test/retest } & \multicolumn{3}{|c|}{ Model's coefficients } & \multirow{2}{*}{$\mathrm{R}^{2}$} \\
\hline & & $\mathrm{B} 2$ & B3 & B4 & \\
\hline \multirow{4}{*}{ D4 } & 1 & $0.875 \pm 1.429$ & $10.965 \pm 1.899$ & $14.741 \pm 1.577$ & 0.485 \\
\hline & 2 & $0.718 \pm 0.190$ & $7.952 \pm 0.619$ & $16.123 \pm 0.634$ & 0.975 \\
\hline & 3 & $0.432 \pm 0.294$ & $11.000 \pm 0.767$ & $16.830 \pm 1.652$ & 0.924 \\
\hline & Averaged & $0.580 \pm 0.337$ & $9.913 \pm 0.891$ & $16.018 \pm 1.210$ & 0.907 \\
\hline \multirow{4}{*}{ D6 } & 1 & $0.749 \pm 0.105$ & $6.984 \pm 0.390$ & $16.465 \pm 0.380$ & 0.993 \\
\hline & 2 & $0.965 \pm 0.064$ & $7.982 \pm 0.171$ & $16.208 \pm 0.130$ & 0.998 \\
\hline & 3 & $1.045 \pm 0.233$ & $10.044 \pm 0.405$ & $15.813 \pm 0.291$ & 0.980 \\
\hline & Averaged & $0.884 \pm 0.043$ & $8.342 \pm 0.117$ & $16.152 \pm 0.096$ & 0.999 \\
\hline \multirow{4}{*}{ D7 } & 1 & $0.385 \pm 0.344$ & $6.000 \pm 1.657$ & $16.876 \pm 4.353$ & 0.885 \\
\hline & 2 & $0.430 \pm 0.286$ & $6.000 \pm 1.388$ & $16.856 \pm 3.017$ & 0.930 \\
\hline & 3 & $0.385 \pm 0.281$ & $5.000 \pm 1.405$ & $16.272 \pm 3.687$ & 0.923 \\
\hline & Averaged & $0.378 \pm 0.186$ & $5.670 \pm 0.930$ & $16.960 \pm 2.520$ & 0.965 \\
\hline \multirow{4}{*}{ D13 } & 1 & $0.140 \pm 1.064$ & $10.000 \pm 1.512$ & $15.061 \pm 27.791$ & 0.407 \\
\hline & 2 & $0.272 \pm 0.283$ & $9.750 \pm 0.765$ & $16.428 \pm 3.818$ & 0.899 \\
\hline & 3 & $0.331 \pm 0.266$ & $9.000 \pm 0.896$ & $16.816 \pm 3.088$ & 0.915 \\
\hline & Averaged & $0.271 \pm 0.146$ & $9.67 \pm 0.360$ & $15.730 \pm 1.790$ & 0.973 \\
\hline
\end{tabular}

Table 11. Levels of short-term memory in drug addicts, females.

\begin{tabular}{cccc}
\hline Level of short-term memory & ID & B3 & B3 cut-off value \\
\hline "extremely below average" & - & - & $<2.295$ \\
"substantially below average" & - & - & $\geq 2.295$ and $<4.742$ \\
"below average" & D7_3 & 5.000 & $\geq 4.742$ and $<6.664$ \\
"average" & D7_1 & 6.000 & \\
& D7_2 & 6.000 & $\geq 6.664$ and $<9.407$ \\
"above average" & D6_1 & 6.984 & \\
& D4_2 & 7.952 & $\geq 9.407$ and $<10.842$ \\
& D6_2 & 7.982 & \\
& D13_3 & 9.000 & \\
& D13_2 & 9.750 & \\
& D13_1 & 10.000 & \\
\hline
\end{tabular}




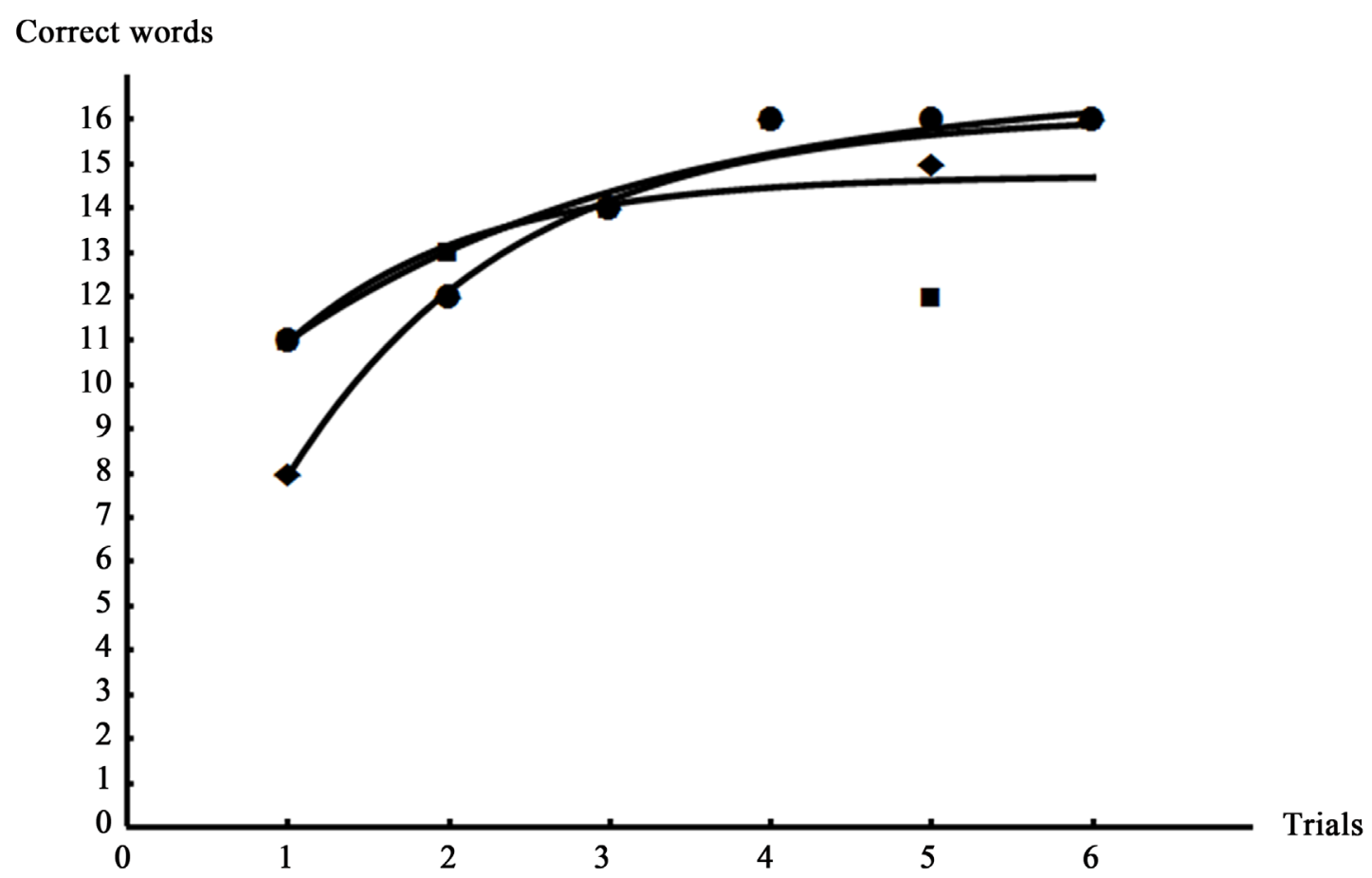

Figure 16. Drug addicts, female with ID $=$ D4. Squares-the first test, diamonds-the second test, circles-the third test.

Drug addicts, female with ID $=$ D6

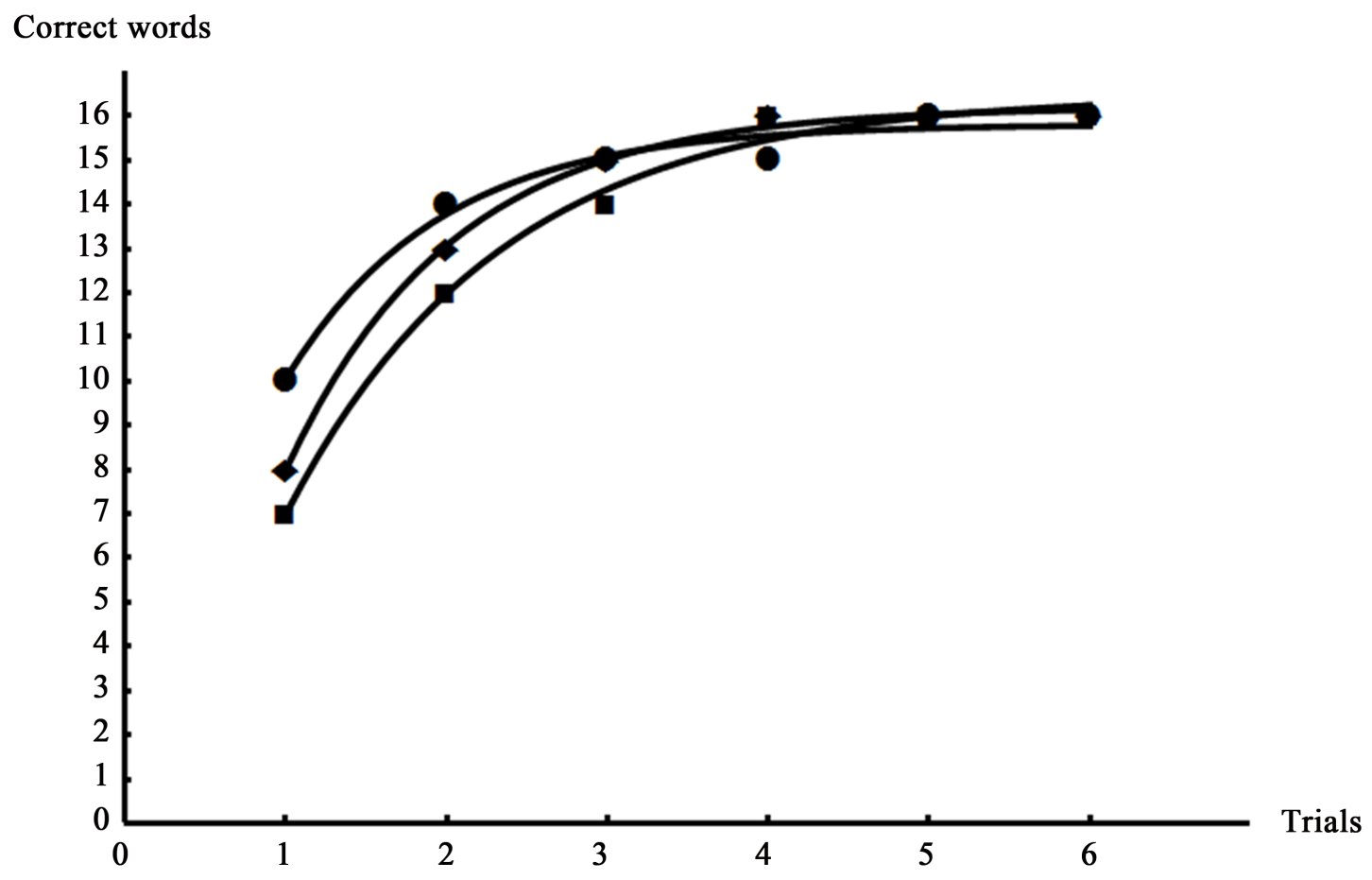

Figure 17. Drug addicts, female with ID = D6. Squares-the first test, diamonds-the second test, circles-the third test. 


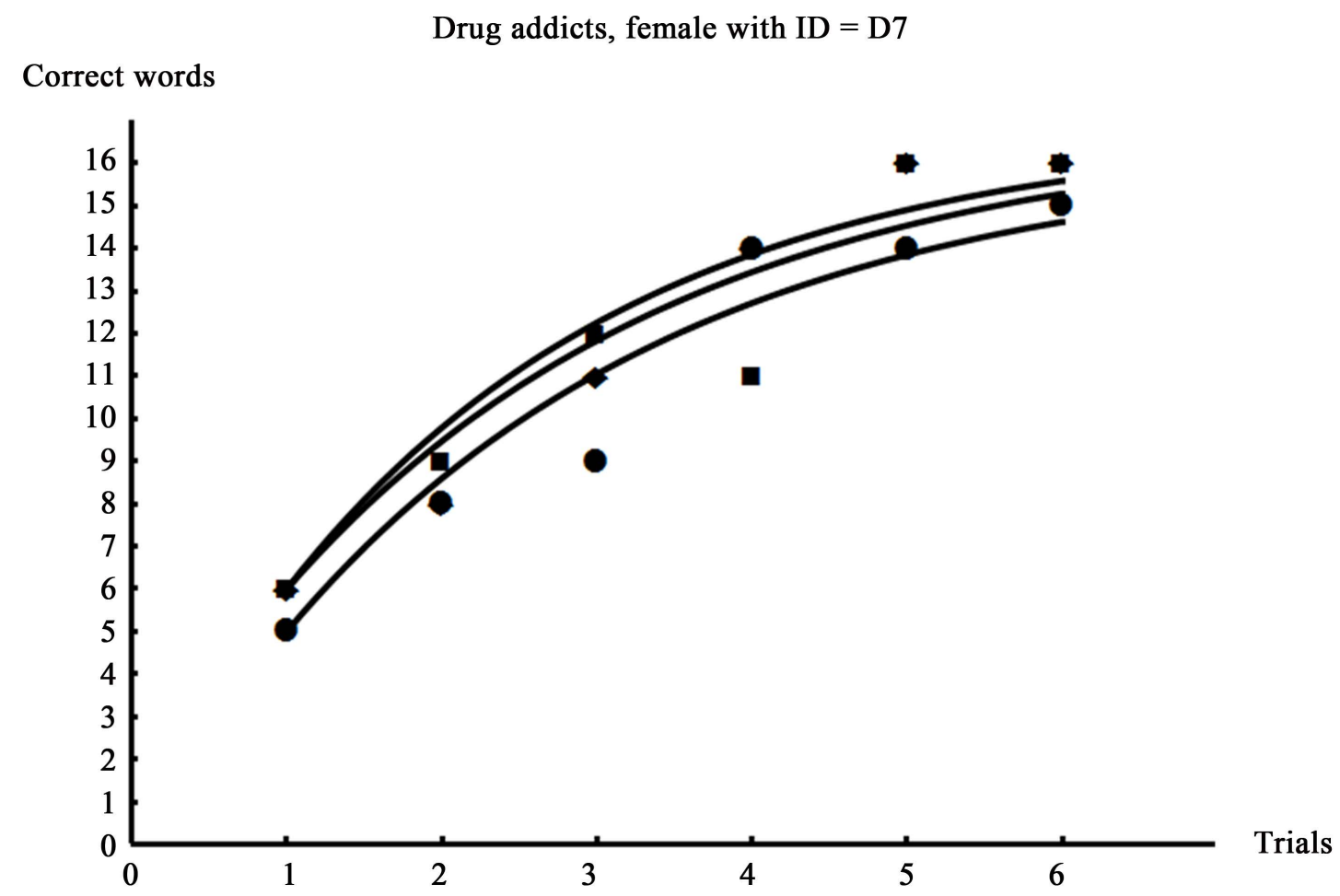

Figure 18. Drug addicts, female with ID $=$ D7. Squares-the first test, diamonds-the second test, circles-the third test.

Drug addicts, female with ID $=$ D13

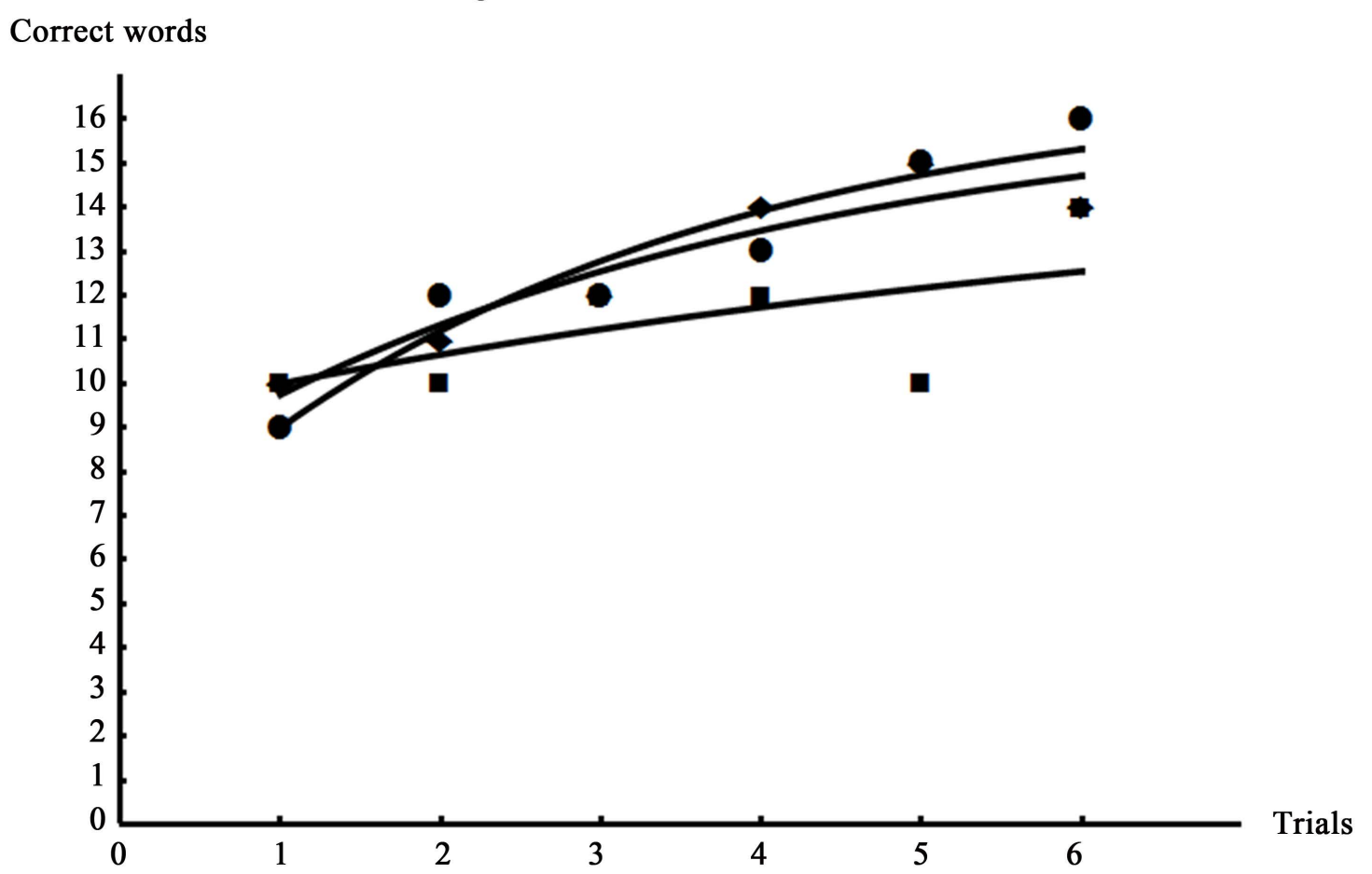

Figure 19. Drug addicts, female with ID = D13. Squares-the first test, diamonds-the second test, circles-the third test. 
Table 12. Levels of long-term memory in drug addicts, females.

\begin{tabular}{cccc}
\hline Level of long-term memory & ID & B4 & B4 cut-off value \\
\hline "extremely below average" & - & - & $<5.692$ \\
"substantially below average" & - & - & $\geq 5.692$ and $<6.124$ \\
"below average" & - & - & $\geq 124$ and $<12.235$ \\
"average" & - & - & $\geq 13.939$ and $<15.1$ \\
"above average" & D4_1 & 14.741 & \\
"well above average" & D13_1 & 15.061 & \\
& D6_3 & 15.813 & \\
& D4_2 & 16.123 & \\
& D6_2 & 16.208 & \\
& D7_3 & 16.272 & \\
& D13_2 & 16.428 & \\
& D6_1 & 16.465 & \\
& D13_3 & 16.816 & \\
& D4_3 & 16.830 & \\
& D7_2 & 16.856 & \\
& D7_1 & 16.876 & \\
& &
\end{tabular}

It is seen from Table 12 that two B4 values were assigned to the "above average" level and ten B4 values to the "well above average" level. This means that each drug addicted female recalled all the words from the list. Thus, in these patients, drug abuse has not yet led to long-term memory impairment.

\section{Drug addicted males}

Raw learning data are provided in Table 13 and the model's coefficients for individual learning curves are provided in Table 14.

Figures below show three individual learning curves for each participant (see Figures 20-30).

Here we see that in each drug addicted male the learning curves differ from each other much more than those of the healthy male participants. A characteristic feature of these learning curves is the large scatter of the trial values around the curve. Instead of a monotonous increase in trial values as the test continues, often these values decrease in the middle of the test-a concave curve or a convexity in the middle of the test, a convex curve, or even an S-shaped curve. Such a deviation in the location of points is a consequence of memory impairment under the influence of drug abuse. Thus, averaging over three tests is even more necessary in drug addicts than in healthy participants.

Analysis of short-term memory levels is presented in Table 15 and long-term memory levels in Table 16.

Table 15 reveals that two B3 values were assigned to the "substantially below average" level, 15 B3 values were assigned to the "below average" level, $11 \mathrm{~B} 3$ values were assigned to the "average" level, three B3 values were assigned to the "above average" level, and two B3 values were assigned to the "well above average" level. This means that patients assigned to "substantially below average" and "below average" levels-D1, D2, D3, D5, D9, D10, D12, and D15 suffer severe short-term memory loss. Thus, short-term memory is partially impaired under the influence of drugs in eight patients. 
Table 13. Raw learning data for drug addicts, males.

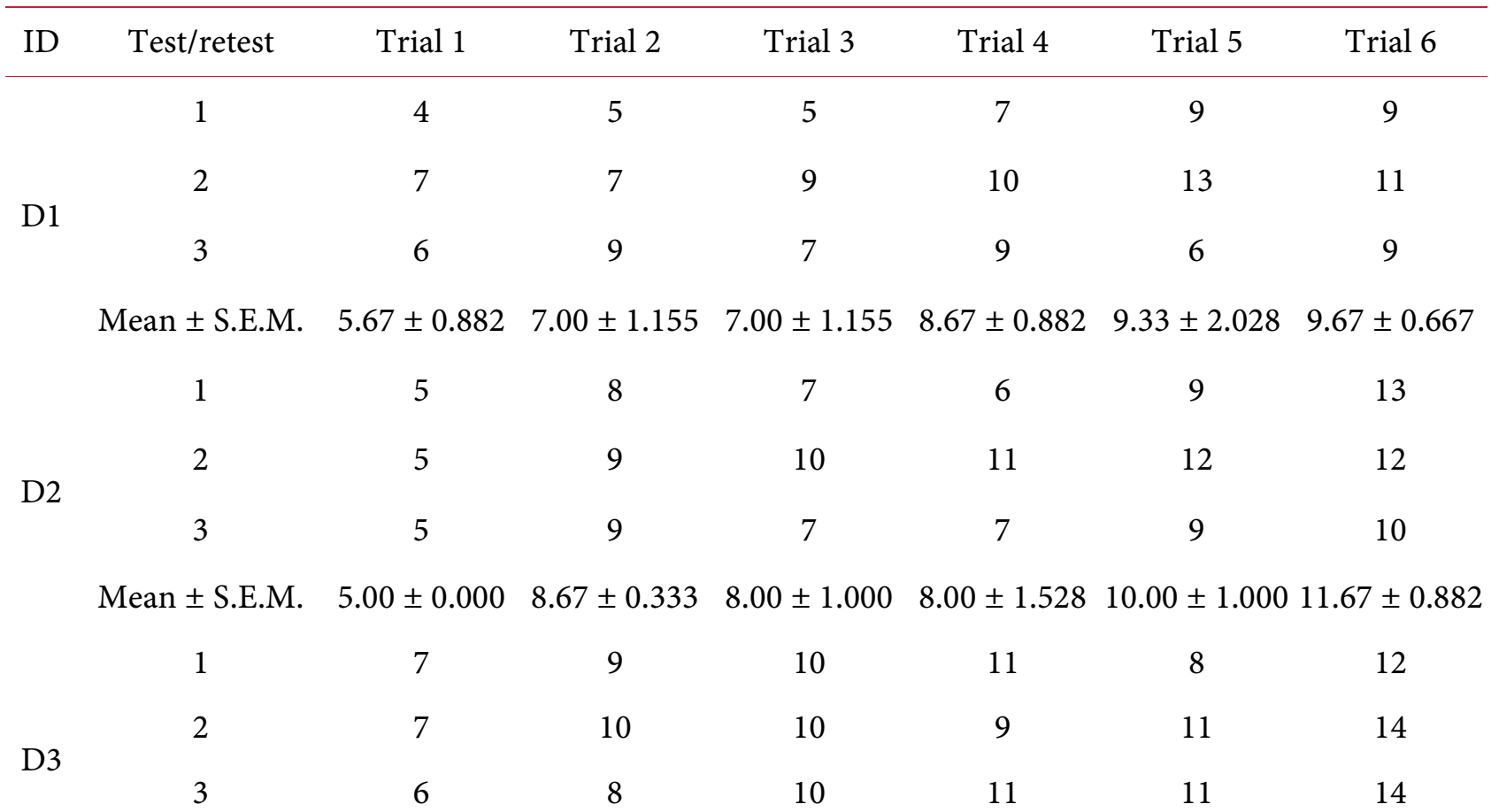

Mean \pm S.E.M. $\quad 6.67 \pm 0.333 \quad 9.00 \pm 0.577 \quad 10.00 \pm 0.00010 .33 \pm 0.66710 .00 \pm 1.00013 .33 \pm 0.667$

$\begin{array}{rlllllll} & 1 & 6 & 6 & 9 & 10 & 12 & 12 \\ \text { D5 } & 2 & 6 & 7 & 9 & 11 & 12 & 14 \\ & 3 & 7 & 7 & 9 & 10 & 13 & 15\end{array}$

Mean \pm S.E.M. $\quad 6.33 \pm 0.333 \quad 6.67 \pm 0.333 \quad 9.00 \pm 0.000 \quad 10.33 \pm 0.33312 .33 \pm 0.33313 .67 \pm 0.882$

$\begin{array}{cccccccc} & 1 & 9 & 8 & 10 & 15 & 15 & 16 \\ \mathrm{D} 8 & 2 & 7 & 10 & 15 & 16 & 16 & 16 \\ & 3 & 8 & 11 & 14 & 16 & 16 & 16\end{array}$

Mean \pm S.E.M. $\quad 8.00 \pm 0.577 \quad 9.67 \pm 0.882 \quad 13.00 \pm 1.52815 .67 \pm 0.33315 .67 \pm 0.33316 .00 \pm 0.000$

$\begin{array}{cccccccc} & 1 & 6 & 8 & 10 & 14 & 13 & 9 \\ \text { D9 } & 2 & 5 & 8 & 6 & 7 & 8 & 12 \\ & 3 & 6 & 9 & 10 & 11 & 8 & 11\end{array}$

Mean \pm S.E.M. $\quad 5.67 \pm 0.333 \quad 8.33 \pm 0.333 \quad 8.67 \pm 1.333 \quad 10.67 \pm 2.028 \quad 9.67 \pm 1.667 \quad 10.67 \pm 0.882$

\begin{tabular}{|c|c|c|c|c|c|c|}
\hline 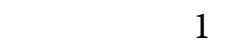 & 6 & 10 & 9 & 10 & 9 & 10 \\
\hline 2 & 5 & 9 & 9 & 10 & 11 & 12 \\
\hline 3 & 7 & 8 & 7 & 9 & 10 & \\
\hline
\end{tabular}

Mean \pm S.E.M. $\quad 6.00 \pm 0.577 \quad 9.00 \pm 0.577 \quad 8.33 \pm 0.667 \quad 9.67 \pm 0.333 \quad 10.00 \pm 0.577 \quad 11.00 \pm 0.577$ 
Continued

\begin{tabular}{cccccccc}
\hline & 1 & 8 & 10 & 11 & 13 & 15 & 15 \\
$\mathrm{D} 11$ & 2 & 7 & 9 & 12 & 14 & 16 & 16 \\
& 3 & 9 & 10 & 9 & 15 & 16 & 16
\end{tabular}

Mean \pm S.E.M. $\quad 8.00 \pm 0.577 \quad 9.67 \pm 0.333 \quad 10.67 \pm 0.88214 .00 \pm 0.57715 .67 \pm 0.33315 .67 \pm 0.333$

$\begin{array}{rlllllll} & 1 & 6 & 7 & 11 & 14 & 13 & 13 \\ \mathrm{D} 12 & 2 & 5 & 8 & 11 & 13 & 15 & 16 \\ & 3 & 6 & 7 & 10 & 13 & 13 & 15\end{array}$

Mean \pm S.E.M. $\quad 5.67 \pm 0.333 \quad 7.33 \pm 0.333 \quad 10.67 \pm 0.33313 .33 \pm 0.33313 .67 \pm 0.66714 .67 \pm 0.882$

$\begin{array}{rccccccc} & 1 & 10 & 12 & 11 & 13 & 14 & 16 \\ \text { D14 } & 2 & 10 & 11 & 10 & 13 & 15 & 16 \\ & 3 & 9 & 10 & 12 & 14 & 16 & 16\end{array}$

Mean \pm S.E.M. $\quad 9.67 \pm 0.33311 .00 \pm 0.57711 .00 \pm 0.57713 .33 \pm 0.33315 .00 \pm 0.57716 .00 \pm 0.000$

\begin{tabular}{|c|c|c|c|c|c|}
\hline 1 & 3 & 10 & 10 & 12 & 12 \\
\hline 2 & 6 & 10 & 11 & 13 & 13 \\
\hline 3 & 8 & 9 & 12 & 14 & 15 \\
\hline
\end{tabular}

Mean \pm S.E.M. $\quad 5.67 \pm 1.453 \quad 9.67 \pm 0.333 \quad 11.00 \pm 0.57713 .00 \pm 0.57713 .33 \pm 0.88215 .00 \pm 0.577$

Correct words

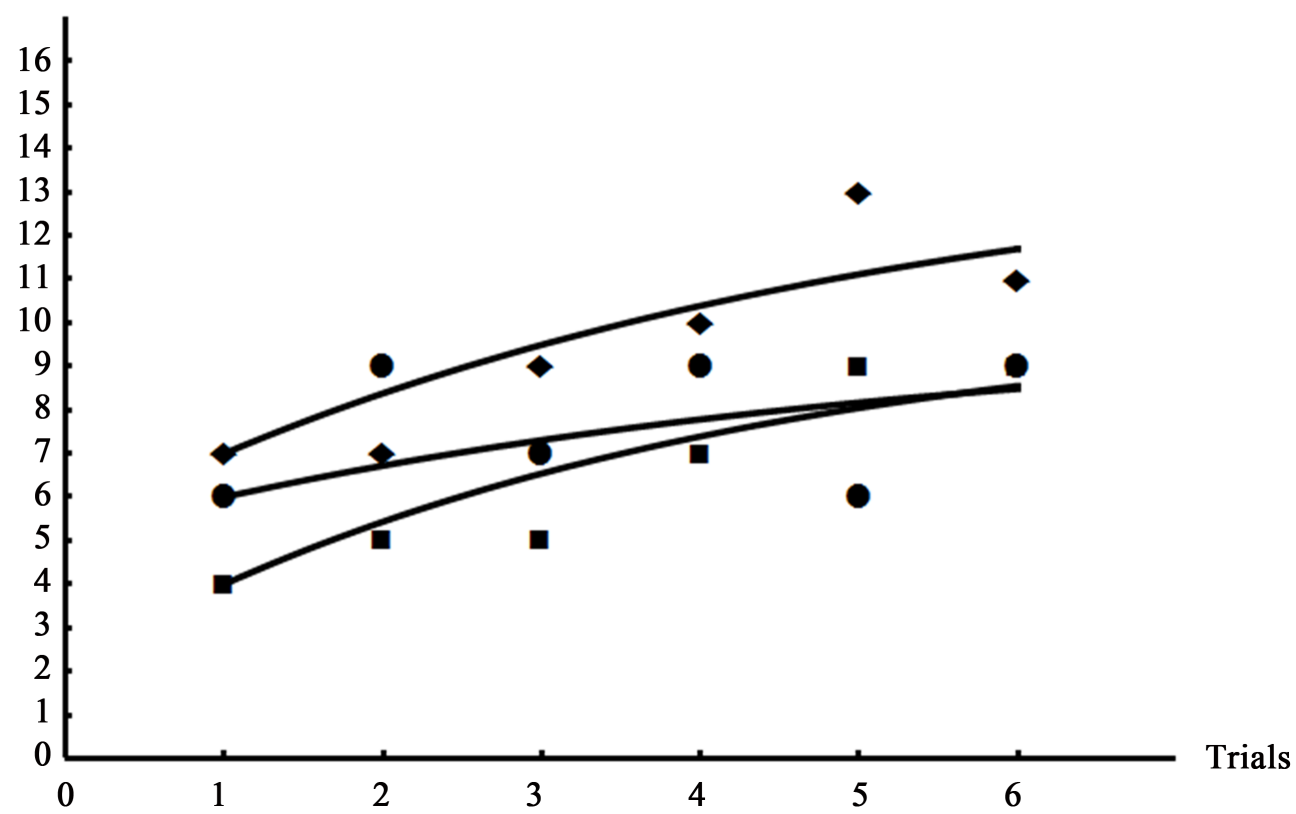

Figure 20. Drug addicts, male with ID = D1. Squares-the first test, diamonds-the second test, circles-the third test. 
Table 14. Individual learning curves for drug addicts, males.

\begin{tabular}{|c|c|c|c|c|c|}
\hline \multirow{2}{*}{ ID } & \multirow{2}{*}{ Test/retest } & \multicolumn{3}{|c|}{ Model's coefficients } & \multirow{2}{*}{$\mathrm{R}^{2}$} \\
\hline & & B2 & B3 & B4 & \\
\hline \multirow{4}{*}{ D1 } & 1 & $0.262 \pm 0.432$ & $4.000 \pm 1.073$ & $10.232 \pm 5.731$ & 0.837 \\
\hline & 2 & $0.216 \pm 0.528$ & $7.000 \pm 1.372$ & $14.109 \pm 10.629$ & 0.769 \\
\hline & 3 & $0.208 \pm 1.356$ & $6.000 \pm 1.863$ & $9.843 \pm 15.625$ & -0.036 \\
\hline & Averaged & $0.094 \pm 0.175$ & $5.711 \pm 0.403$ & $16.661 \pm 16.312$ & 0.954 \\
\hline \multirow{4}{*}{$\mathrm{D} 2$} & 1 & $0.177 \pm 0.754$ & $5.000 \pm 2.283$ & $14.256 \pm 26.095$ & 0.555 \\
\hline & 2 & $0.674 \pm 0.132$ & $5.103 \pm 0.385$ & $12.206 \pm 0.426$ & 0.987 \\
\hline & 3 & $0.279 \pm 0.677$ & $5.000 \pm 1.645$ & $10.949 \pm 7.791$ & 0.467 \\
\hline & Averaged & $0.283 \pm 0.425$ & $5.00 \pm 1.34$ & $12.69 \pm 6.20$ & 0.762 \\
\hline \multirow{4}{*}{ D3 } & 1 & $0.873 \pm 1.375$ & $7.008 \pm 1.689$ & $10.492 \pm 1.406$ & 0.503 \\
\hline & 2 & $0.221 \pm 0.539$ & $7.000 \pm 1.610$ & $15.098 \pm 11.956$ & 0.674 \\
\hline & 3 & $0.280 \pm 0.242$ & $6.000 \pm 0.898$ & $15.100 \pm 4.243$ & 0.930 \\
\hline & Averaged & $0.247 \pm 0.382$ & $6.67 \pm 1.14$ & $14.36 \pm 6.82$ & 0.812 \\
\hline \multirow{4}{*}{ D5 } & 1 & $0.306 \pm 0.393$ & $6.000 \pm 1.202$ & $13.263 \pm 4.810$ & 0.871 \\
\hline & 2 & $0.271 \pm 0.275$ & $6.000 \pm 1.028$ & $15.273 \pm 5.161$ & 0.925 \\
\hline & 3 & $0.215 \pm 0.519$ & $7.000 \pm 1.782$ & $16.422 \pm 13.957$ & 0.797 \\
\hline & Averaged & $0.258 \pm 0.361$ & $6.33 \pm 1.24$ & $14.98 \pm 6.82$ & 0.884 \\
\hline \multirow{4}{*}{ D8 } & 1 & $0.299 \pm 0.725$ & $9.000 \pm 2.369$ & $16.822 \pm 9.879$ & 0.705 \\
\hline & 2 & $0.624 \pm 0.273$ & $7.000 \pm 1.140$ & $16.881 \pm 1.395$ & 0.946 \\
\hline & 3 & $0.564 \pm 0.172$ & $8.000 \pm 0.664$ & $16.869 \pm 0.937$ & 0.975 \\
\hline & Averaged & $0.471 \pm 0.279$ & $8.00 \pm 1.10$ & $16.85 \pm 2.06$ & 0.935 \\
\hline \multirow{4}{*}{ D9 } & 1 & $0.788 \pm 1.041$ & $5.655 \pm 2.452$ & $11.888 \pm 2.260$ & 0.599 \\
\hline & 2 & $0.176 \pm 0.727$ & $5.000 \pm 1.941$ & $13.202 \pm 22.564$ & 0.561 \\
\hline & 3 & $1.518 \pm 1.852$ & $5.983 \pm 1.425$ & $10.030 \pm 0.839$ & 0.676 \\
\hline & Averaged & $0.642 \pm 0.327$ & $5.721 \pm 0.674$ & $10.642 \pm 0.795$ & 0.920 \\
\hline \multirow{4}{*}{ D10 } & 1 & $0.546 \pm 0.670$ & $6.000 \pm 1.335$ & $10.542 \pm 1.980$ & 0.533 \\
\hline & 2 & $0.497 \pm 0.270$ & $5.329 \pm 0.808$ & $12.046 \pm 1.383$ & 0.929 \\
\hline & 3 & $0.191 \pm 0.573$ & $7.000 \pm 1.015$ & $12.179 \pm 9.977$ & 0.737 \\
\hline & Averaged & $0.403 \pm 0.356$ & $6.335 \pm 0.780$ & $11.251 \pm 1.894$ & 0.869 \\
\hline
\end{tabular}




\section{Continued}

\begin{tabular}{|c|c|c|c|c|c|}
\hline \multirow{4}{*}{ D11 } & 1 & $0.345 \pm 0.232$ & $8.000 \pm 0.827$ & $16.208 \pm 2.646$ & 0.944 \\
\hline & 2 & $0.431 \pm 0.238$ & $7.000 \pm 1.047$ & $16.841 \pm 2.261$ & 0.949 \\
\hline & 3 & $0.330 \pm 0.716$ & $9.000 \pm 2.407$ & $16.822 \pm 8.358$ & 0.692 \\
\hline & Averaged & $0.341 \pm 0.322$ & $8.00 \pm 1.25$ & $16.98 \pm 4.09$ & 0.904 \\
\hline \multirow{4}{*}{ D12 } & 1 & $0.417 \pm 0.343$ & $5.379 \pm 1.468$ & $14.976 \pm 3.366$ & 0.879 \\
\hline & 2 & $0.406 \pm 0.153$ & $5.000 \pm 0.810$ & $16.893 \pm 1.938$ & 0.976 \\
\hline & 3 & $0.315 \pm 0.287$ & $6.000 \pm 1.255$ & $16.278 \pm 4.747$ & 0.921 \\
\hline & Averaged & $0.381 \pm 0.222$ & $5.67 \pm 1.00$ & $15.89 \pm 2.69$ & 0.952 \\
\hline \multirow{4}{*}{ D14 } & 1 & $0.240 \pm 0.452$ & $10.000 \pm 1.181$ & $16.807 \pm 7.474$ & 0.800 \\
\hline & 2 & $0.238 \pm 0.636$ & $10.000 \pm 1.668$ & $16.865 \pm 10.746$ & 0.722 \\
\hline & 3 & $0.365 \pm 0.333$ & $9.000 \pm 1.146$ & $16.839 \pm 3.320$ & 0.905 \\
\hline & Averaged & $0.275 \pm 0.394$ & $9.67 \pm 1.15$ & $16.89 \pm 5.63$ & 0.860 \\
\hline \multirow{4}{*}{ D15 } & 1 & $0.859 \pm 0.359$ & $3.250 \pm 1.243$ & $12.958 \pm 1.051$ & 0.935 \\
\hline & 2 & $0.393 \pm 0.169$ & $6.238 \pm 0.715$ & $15.748 \pm 1.812$ & 0.967 \\
\hline & 3 & $0.358 \pm 0.268$ & $8.000 \pm 1.041$ & $16.890 \pm 3.124$ & 0.934 \\
\hline & Averaged & $0.388 \pm 0.120$ & $5.857 \pm 0.543$ & $16.041 \pm 1.409$ & 0.983 \\
\hline
\end{tabular}

Correct words

Drug addicts, male with ID $=$ D2

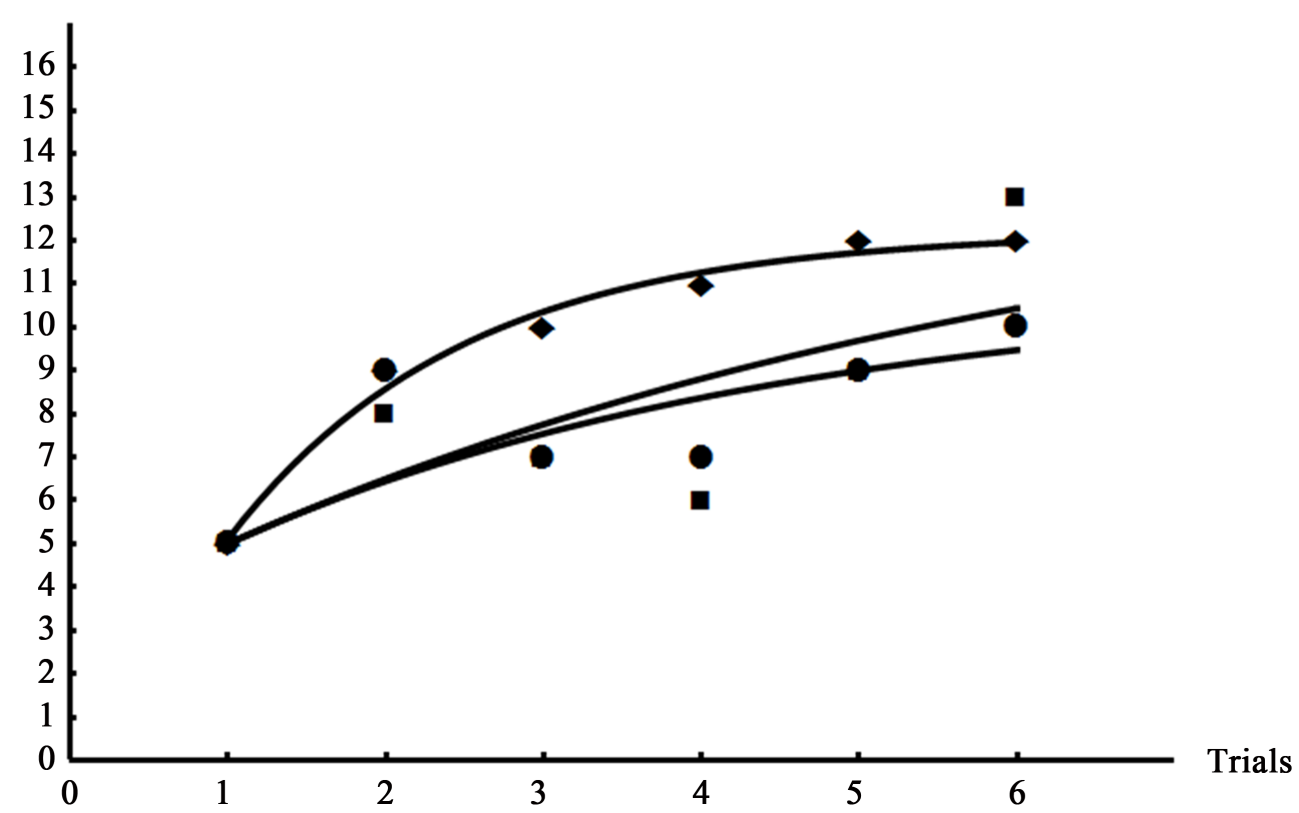

Figure 21. Drug addicts, male with ID = D2. Squares-the first test, diamonds-the second test, circles-the third test. 
Table 15. Levels of short-term memory in drug addicts, males.

\begin{tabular}{|c|c|c|c|}
\hline Level of short-term memory & ID & B3 & B3 cut-off value \\
\hline "extremely below average" & - & - & $<2.292$ \\
\hline \multirow{9}{*}{ "substantially below average" } & D15_1 & 3.250 & \multirow{9}{*}{$\geq 2.292$ and $<4.625$} \\
\hline & $\mathrm{D} 1 \_1$ & 4.000 & \\
\hline & D2_1 & 5.000 & \\
\hline & D2_3 & 5.000 & \\
\hline & D9_2 & 5.000 & \\
\hline & D12_2 & 5.000 & \\
\hline & D2_2 & 5.103 & \\
\hline & D10_2 & 5.329 & \\
\hline & D12_1 & 5.379 & \\
\hline \multirow[t]{13}{*}{ "below average" } & D9_1 & 5.655 & \multirow[t]{13}{*}{$\geq 4.625$ and $<6.190$} \\
\hline & D9_3 & 5.983 & \\
\hline & D1_3 & 6.000 & \\
\hline & D3_3 & 6.000 & \\
\hline & D5_1 & 6.000 & \\
\hline & D5_2 & 6.000 & \\
\hline & D10_1 & 6.000 & \\
\hline & D12_3 & 6.000 & \\
\hline & D15_2 & 6.238 & \\
\hline & D1_2 & 7.000 & \\
\hline & D3_2 & 7.000 & \\
\hline & D5_3 & 7.000 & \\
\hline & D8_2 & 7.000 & \\
\hline \multirow[t]{7}{*}{ "average" } & D10_3 & 7.000 & \multirow[t]{7}{*}{$\geq 6.190$ and $<8.624$} \\
\hline & D11_2 & 7.000 & \\
\hline & D3_1 & 7.008 & \\
\hline & D8_3 & 8.000 & \\
\hline & D11_1 & 8.000 & \\
\hline & D15_3 & 8.000 & \\
\hline & D8_1 & 9.000 & \\
\hline \multirow[t]{2}{*}{ "above average" } & D11_3 & 9.000 & \multirow[t]{2}{*}{$\geq 8.624$ and $<9.996$} \\
\hline & D14_3 & 9.000 & \\
\hline \multirow{2}{*}{ "well above average" } & D14_1 & 10.000 & \multirow{2}{*}{$\geq 9.996$} \\
\hline & D14_2 & 10.000 & \\
\hline
\end{tabular}


Table 16. Levels of long-term memory in drug addicts, males.

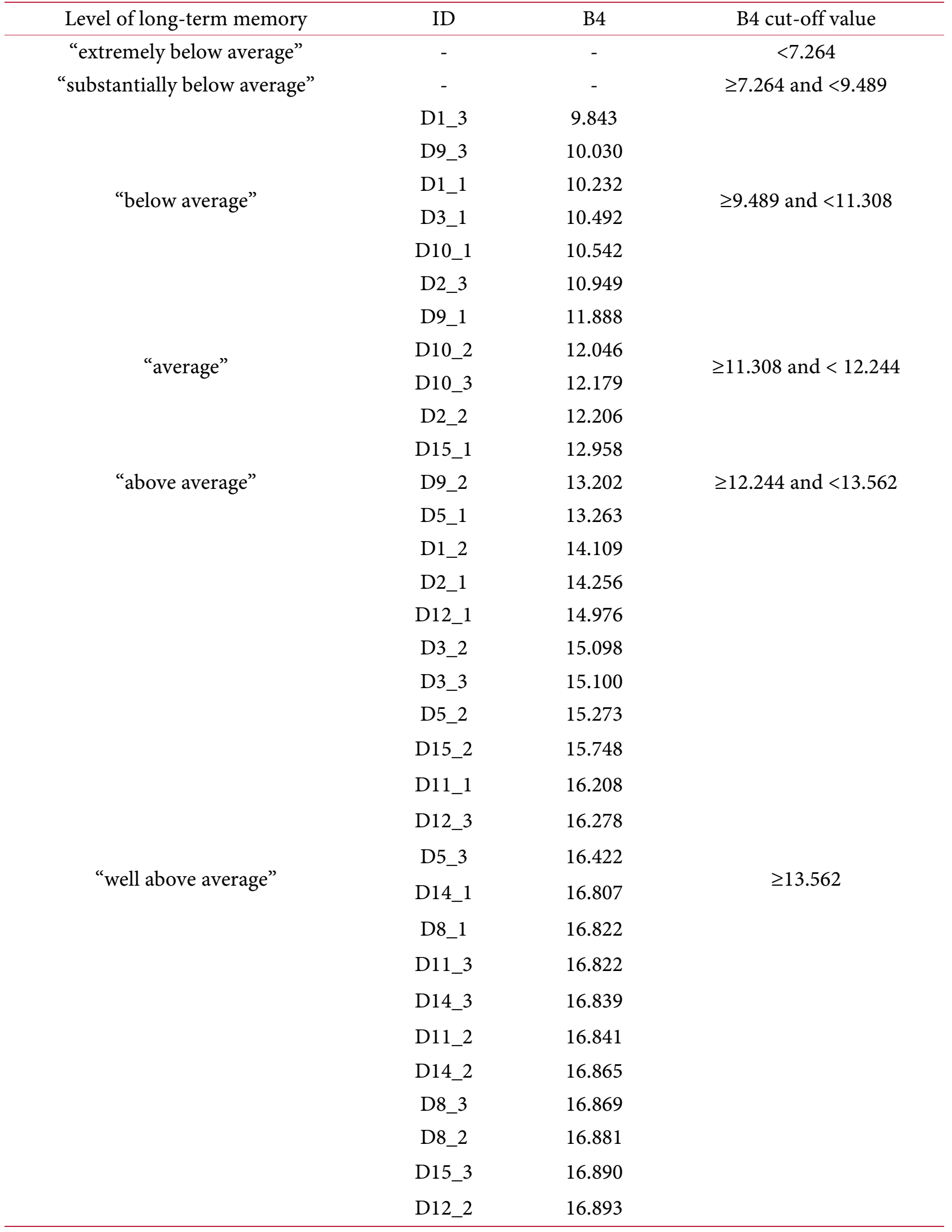


Drug addicts, male with ID $=$ D3

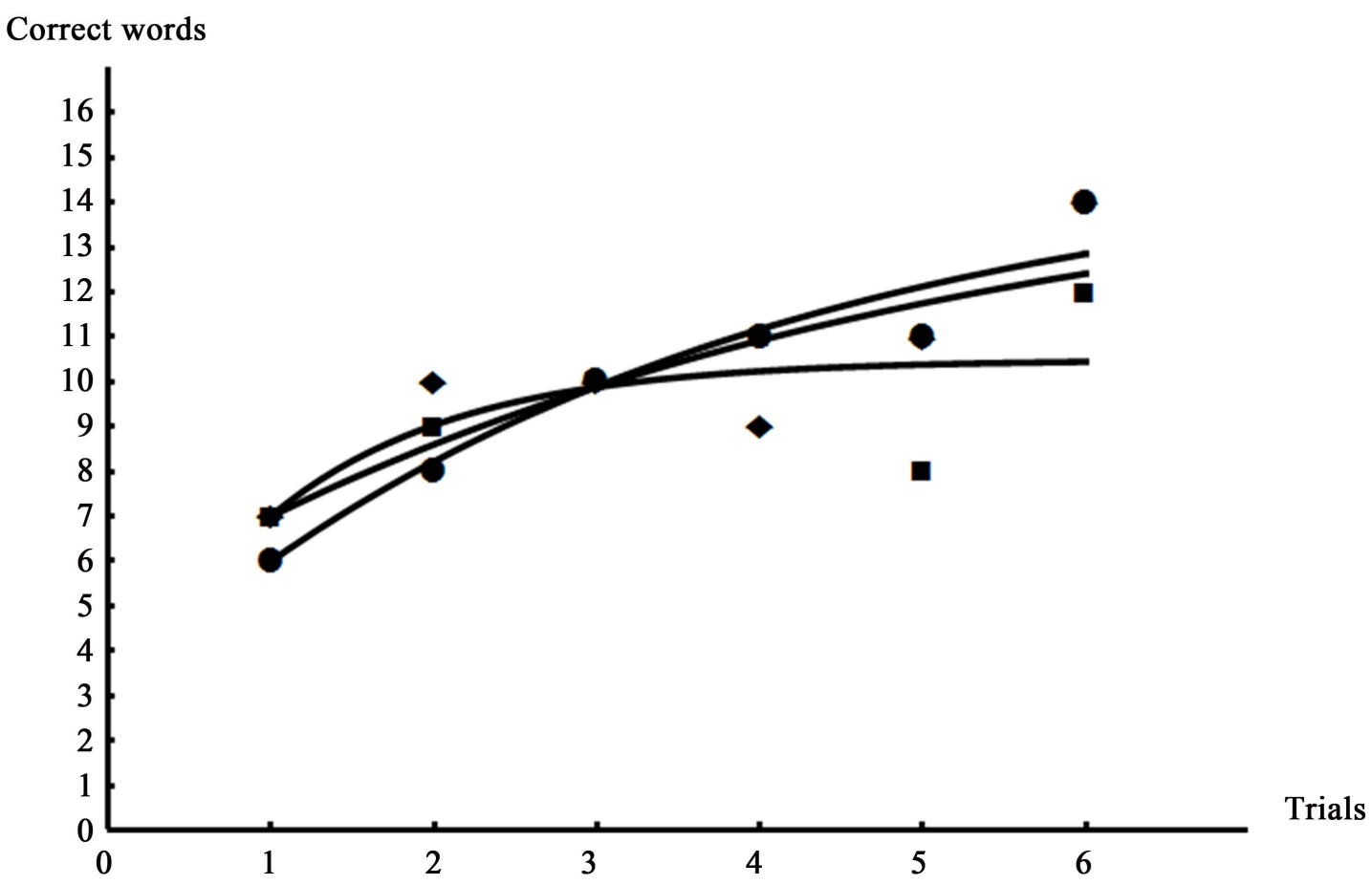

Figure 22. Drug addicts, male with ID = D3. Squares-the first test, diamonds-the second test, circles-the third test.

Drug addicts, male with ID = D5

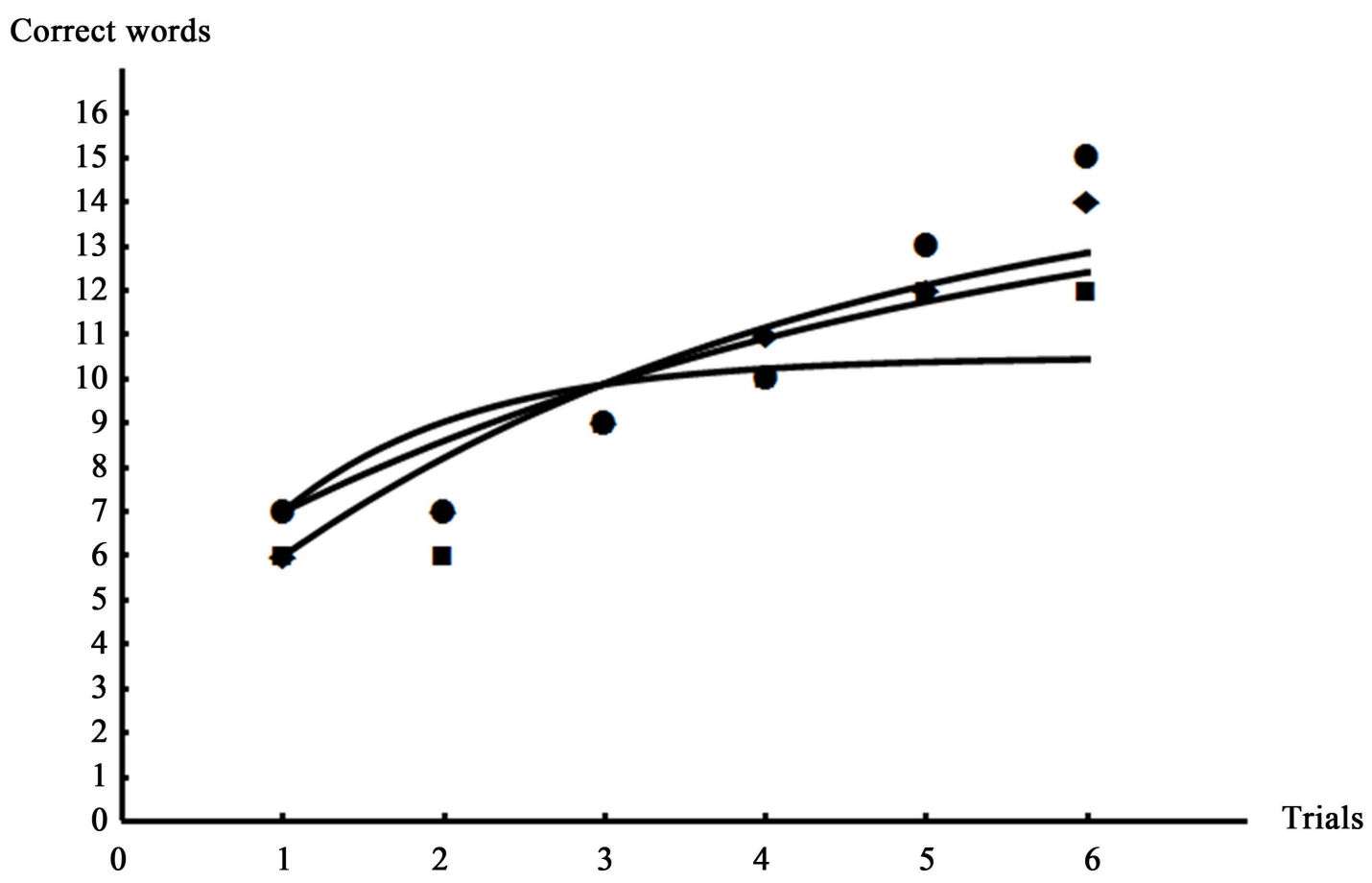

Figure 23. Drug addicts, male with ID = D5. Squares-the first test, diamonds-the second test, circles-the third test. 


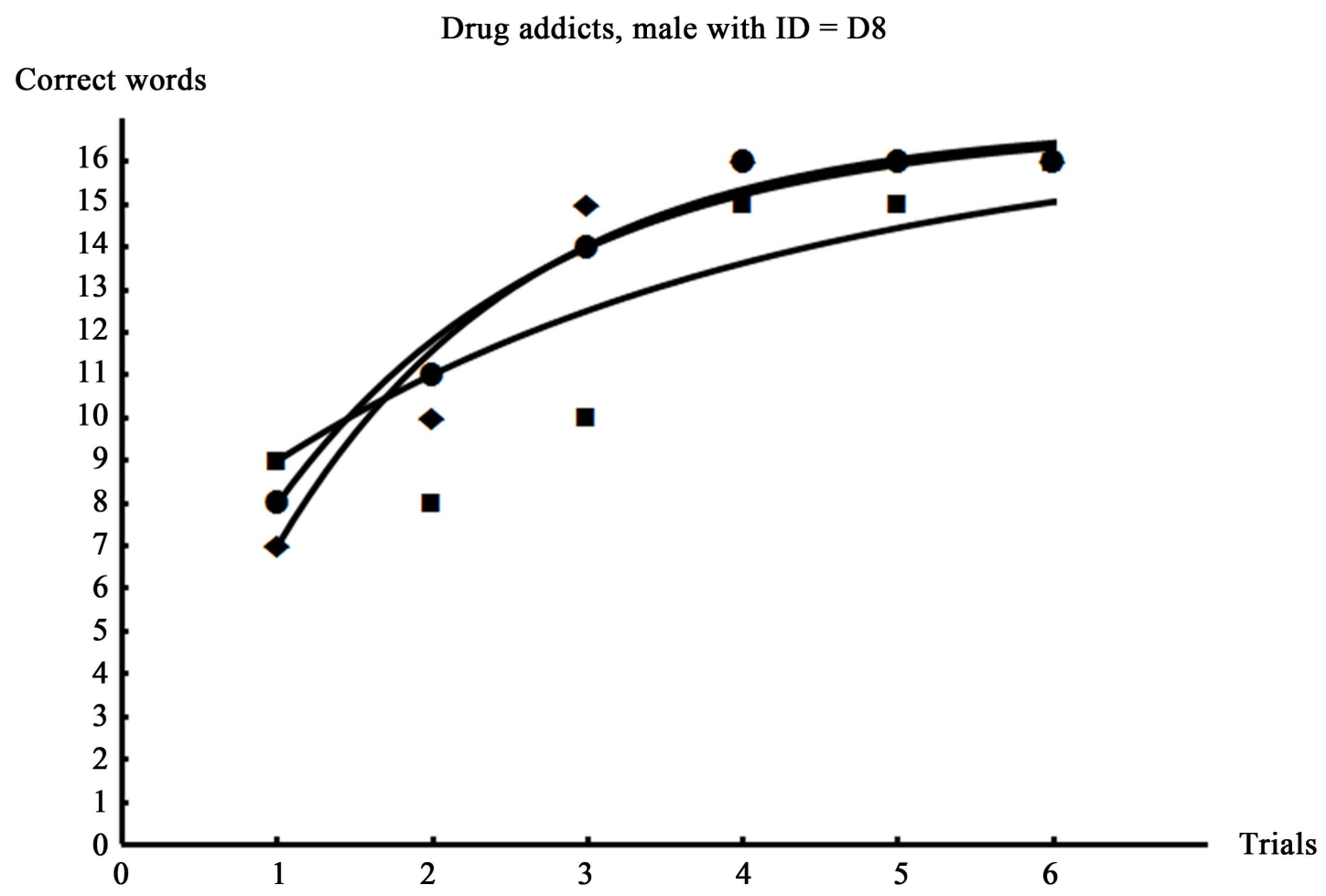

Figure 24. Drug addicts, male with ID $=$ D8. Squares-the first test, diamonds-the second test, circles-the third test.

$$
\text { Drug addicts, male with ID = D9 }
$$

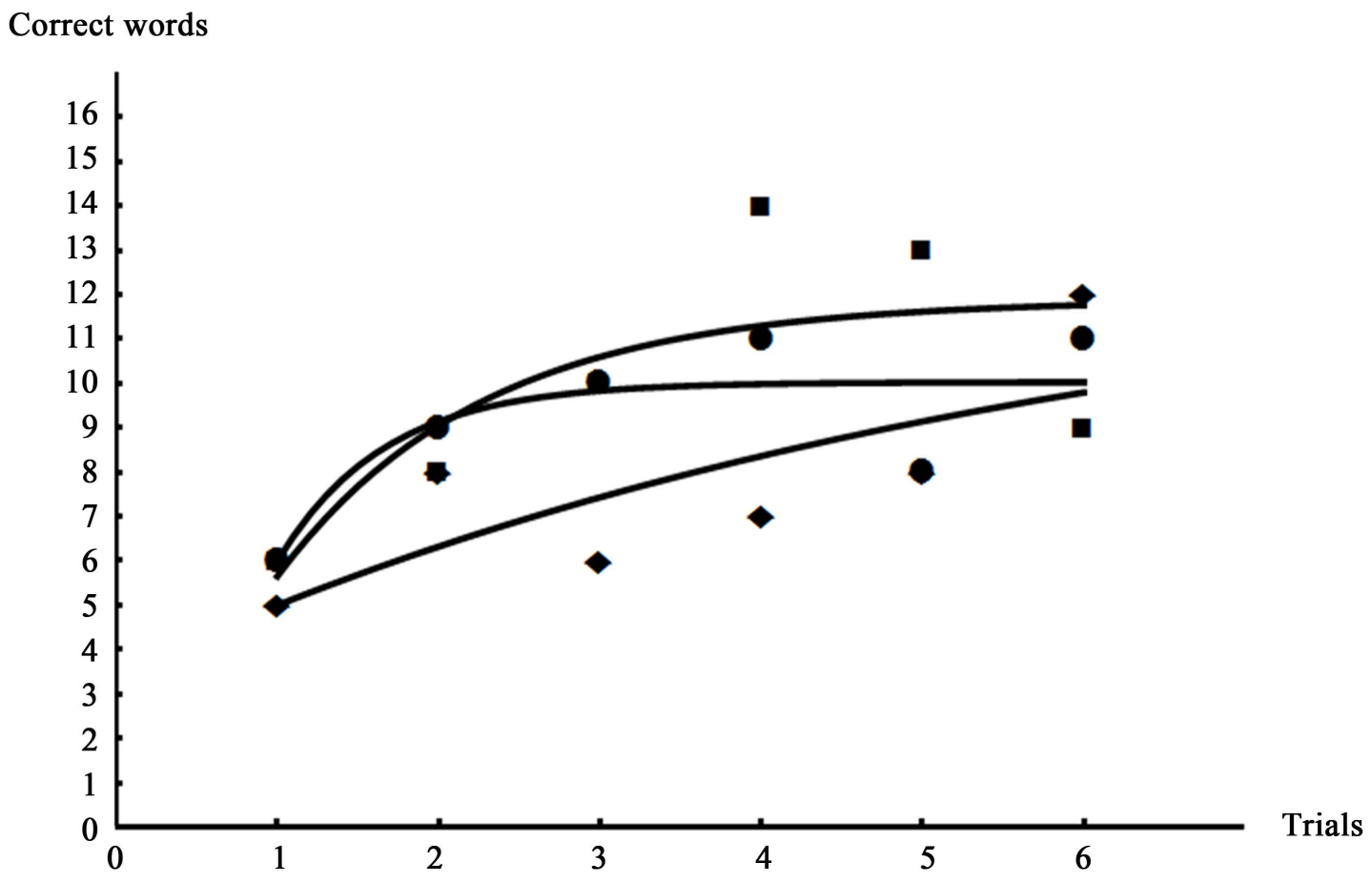

Figure 25. Drug addicts, male with ID = D9. Squares-the first test, diamonds-the second test, circles-the third test. 


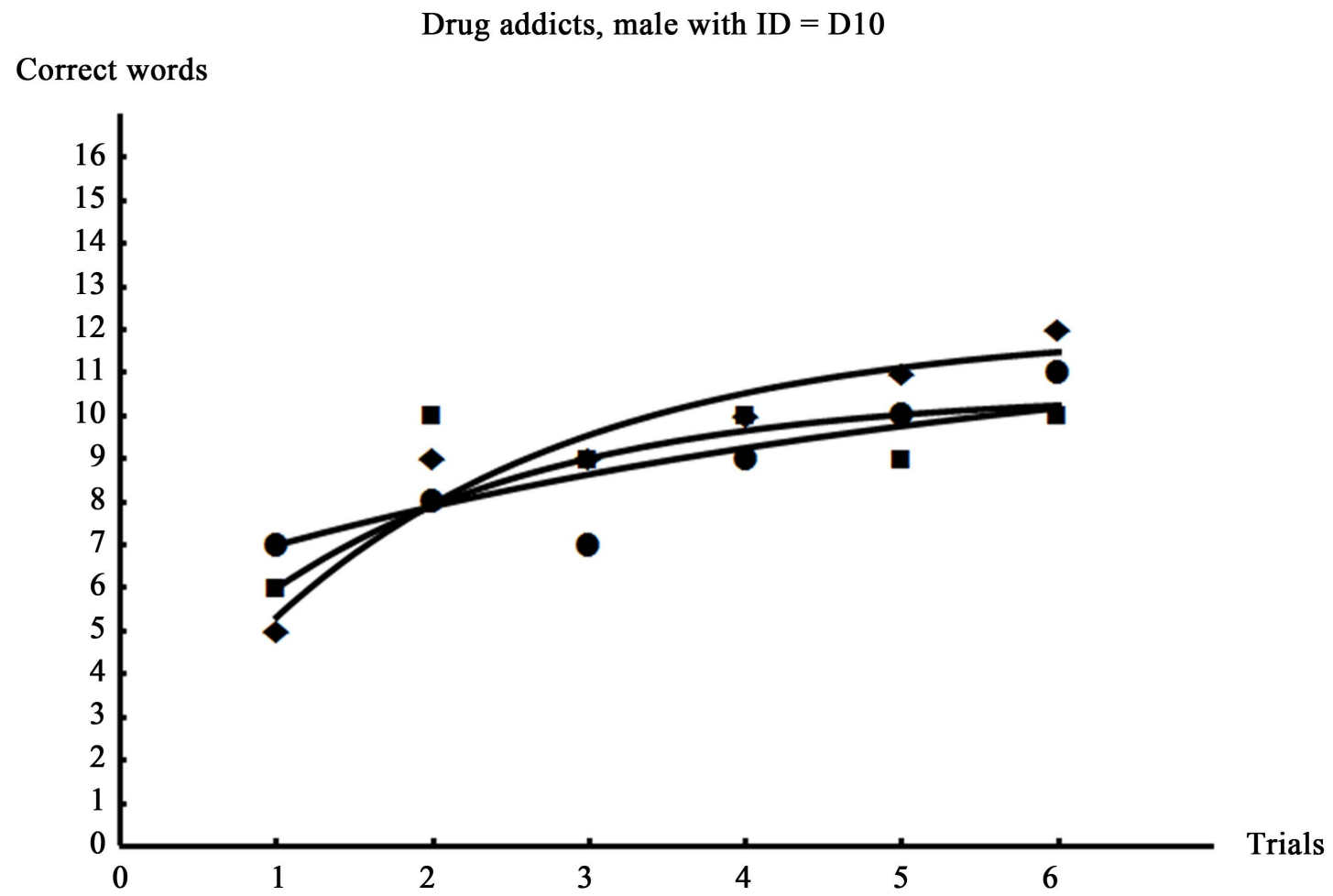

Figure 26. Drug addicts, male with ID = D10. Squares-the first test, diamonds-the second test, circles-the third test.

$$
\text { Drug addicts, male with ID }=\text { D11 }
$$

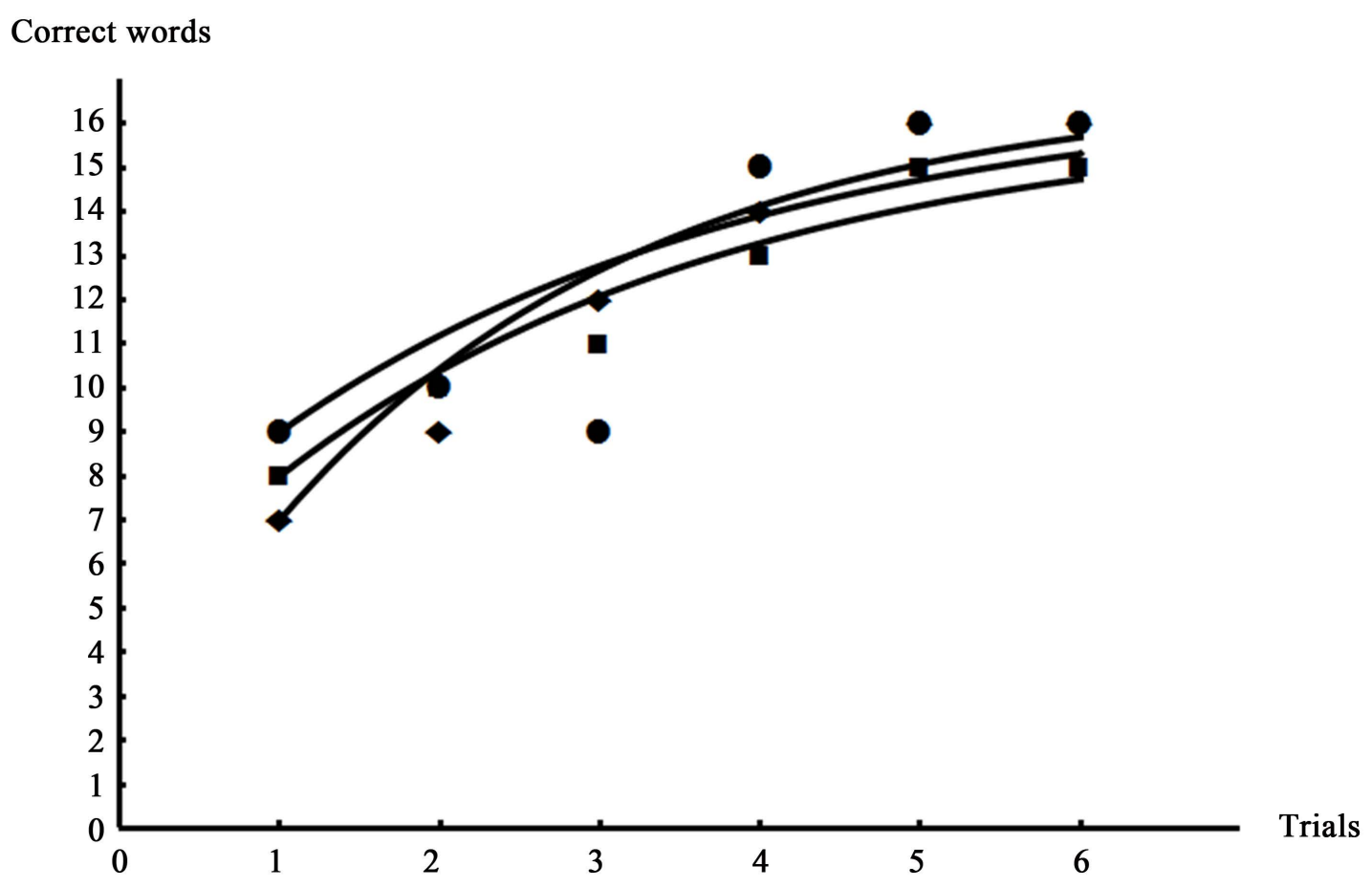

Figure 27. Drug addicts, male with ID = D11. Squares-the first test, diamonds-the second test, circles-the third test. 


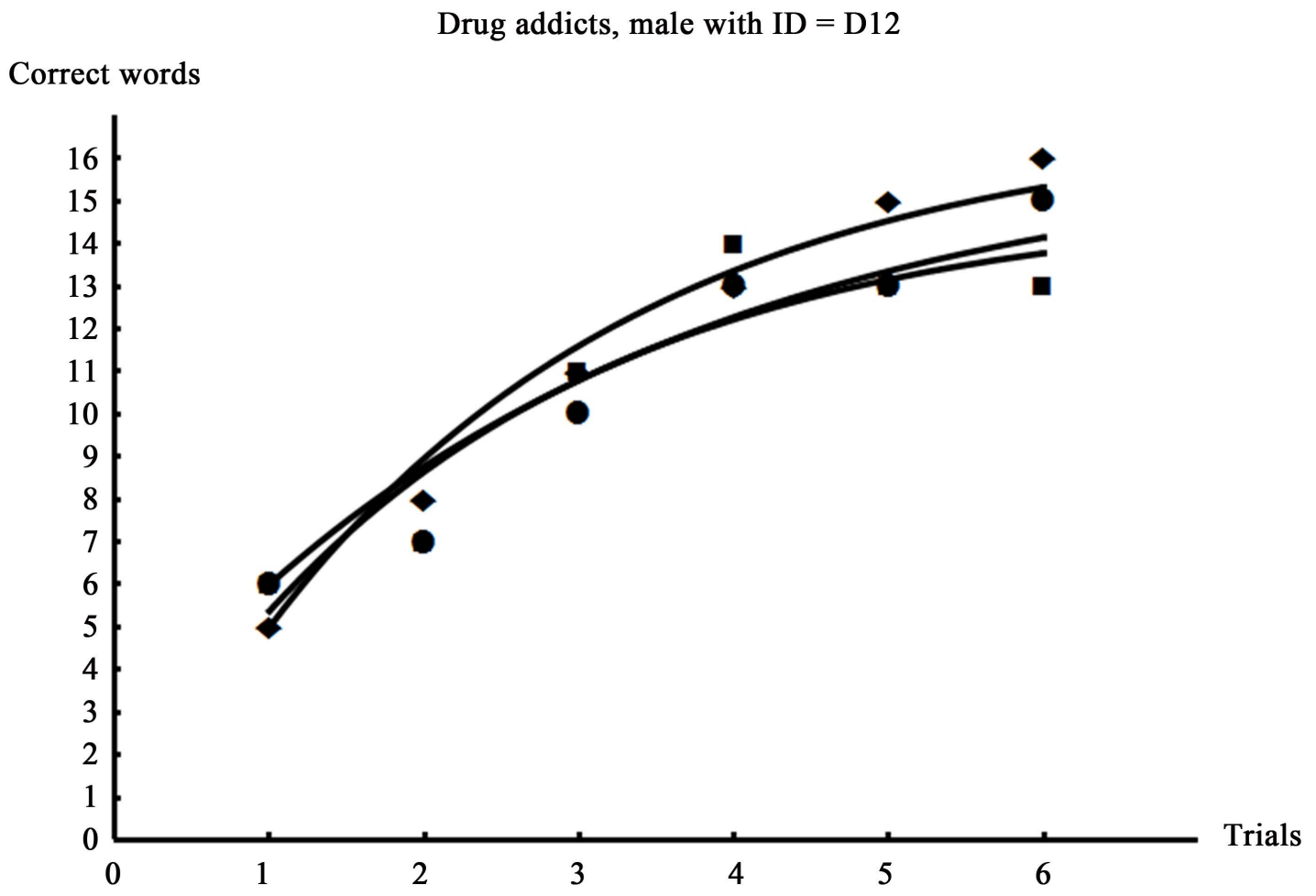

Figure 28. Drug addicts, male with ID = D12. Squares-the first test, diamonds-the second test, circles-the third test.

$$
\text { Drug addicts, male with ID }=\text { D14 }
$$

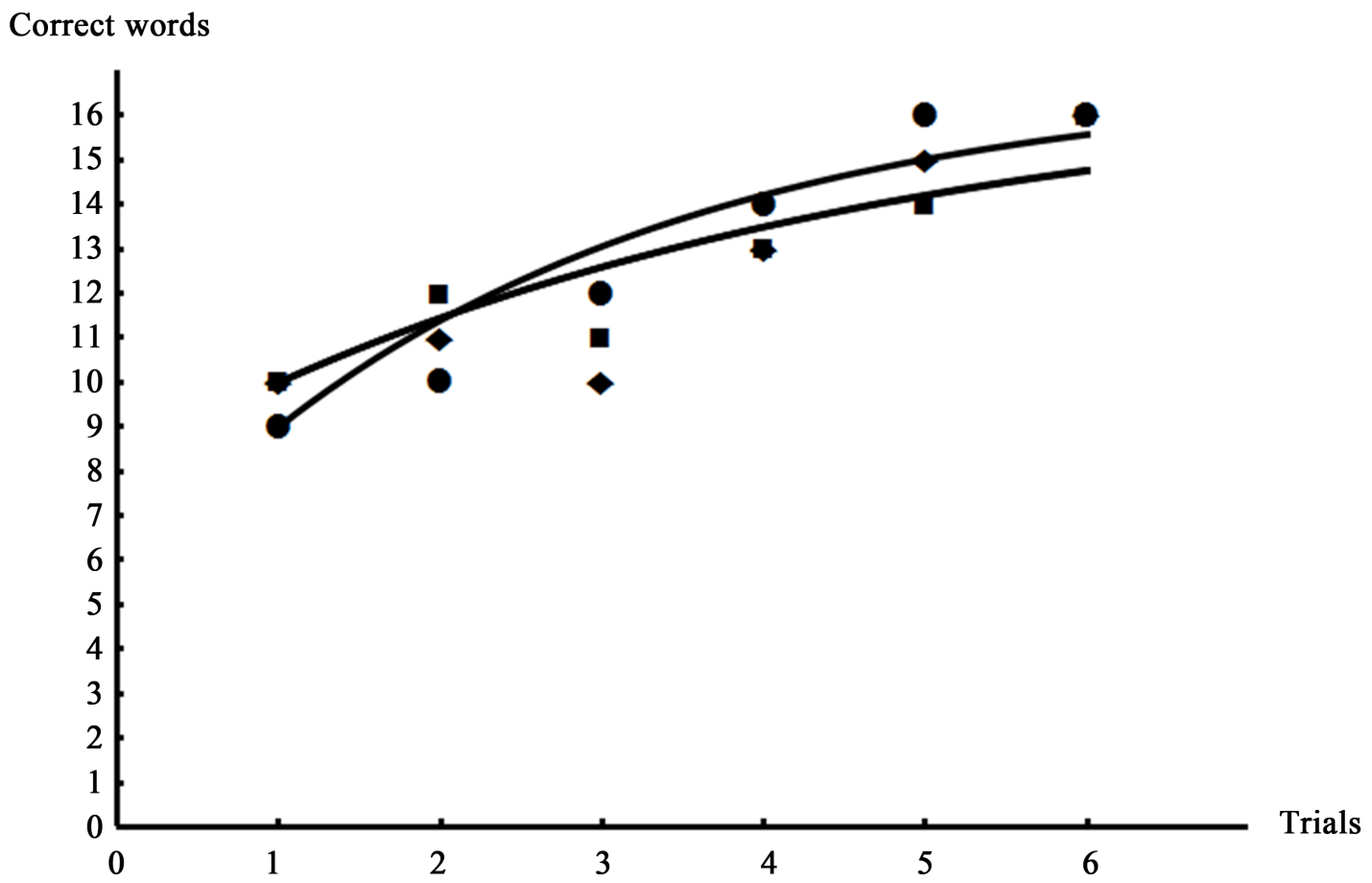

Figure 29. Drug addicts, male with ID = D14. Squares-the first test, diamonds-the second test, circles-the third test. 


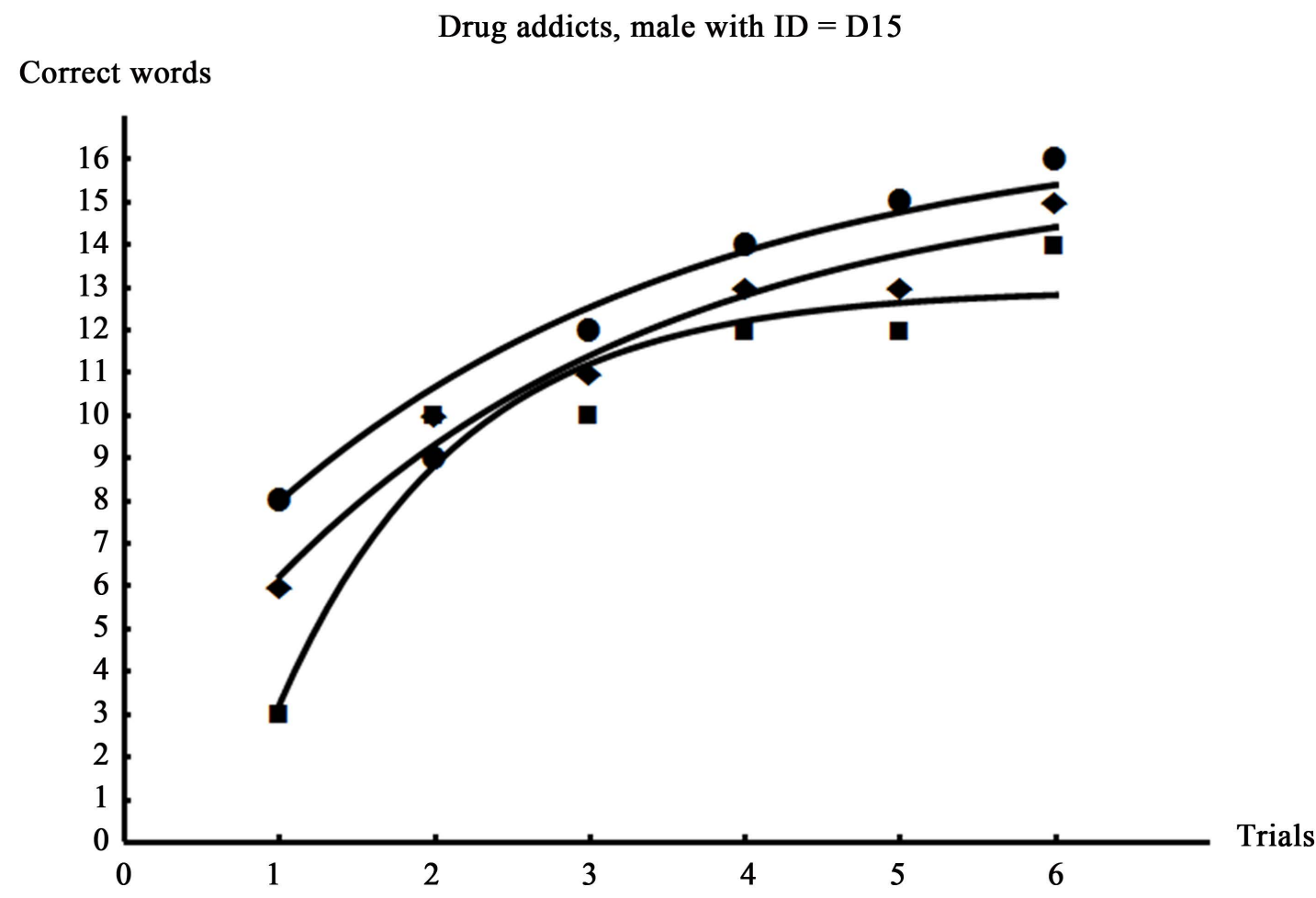

Figure 30. Drug addicts, male with ID = D15. Squares-the first test, diamonds-the second test, circles-the third test.

Table 16 shows that six B4 values were assigned to the "below average" level, four B4 values were assigned to the "average" level, three B4 values were assigned to the "above average" level, and 20 B4 values were assigned to the "well above average" level. This means that patients D1, D2, D3, D9, and D10 exhibit severe deficiency in their long-term memory. Moreover, both short- and long-term memory are impaired in patients D1, D2, D3, D9, and D10. It is necessary to mention that long-term memory was not been impaired in patients D12, D5, D15, D11, D12, D14, and D8. Of particular interest is patient D3, whose first B4 value was assigned to "below average" level, but the next two B4 values were assigned to "well above average" level.

\section{DISCUSSION}

The results presented here show how the model can be used to characterize the effect of marijuana on learning and memory. Although we illustrated our model on individuals using an amphetamine-like psychostimulant drug, the methods and rationale are readily extended to the study of marijuana. A key aspect of our model is that we focus on individual performance which allows the researcher to make a clinical assessment at the level of the individual. The model is applicable for researchers interested in using the CVLT or the memory test developed by the senior authors.

We would like to note that our model can be used to test the claims of any plant based product. Claims have long been made about memory enhancing benefits of various plant-based products. While some claims have failed in scientific studies, curcumin has been shown in animal models to reduce depression by working on the cognitive impairments it causes, including effects on memory and learning [16]. Flavinol in cocoa has been shown to improve memory in invertebrates (snails), as well as spatial memory in rats [17]. Natural and synthetic memory supplements have a sizeable market, and sales were estimated to be $\$ 643$ million in 2015 , with the number of supplements available to consumers growing from 4000 to over 80,000 from 2004 to 2016 [18]. Catechin-rich oil palm leaf extract supplements, for example, have 
been tested on young adults and resulted in increased short-term memory, processing speed, and spatial visual learning after one month [19].

Approximately $15 \%$ of people over the age of 65 suffer from Alzheimer's disease [19]. The implications of studies where memory is improved by natural supplements denote an importance to sort through products with legitimate support for memory enhancement and potential for memory-loss prevention. The mathematical model posed by the authors can be used to do further research on the learning and memory enhancing properties of these plant derivatives in animals, as well as in humans.

An area with an immediate need for research due to concern regarding the negative effects of plant-based products on memory is in the growing field of medical marijuana. In the United States, approximately 30 states allow marijuana for medical purposes, although individual state regulations vary significantly. An increase in the number of medical marijuana users dictates a need to determine the possible side effects of using this substance. Of particular concern are the possible effects of marijuana on neurocognitive functioning, including learning and memory [20,21].

The senior authors' model can easily be incorporated into various experimental designs and supplement traditional statistical tests. In the example shown here, we used a group design where drug free males and females were compared with males and females who regularly consumed an amphetamine-like psychostimulant drug. The model can also be used in "ABA" designs where participants are exposed to marijuana or other plant based products, subsequently exposed, and then no longer exposed.

We recommend people who regularly use marijuana for medical purposes or other plant based products regularly test their memory for identification of early signs of impairment. For those researchers and clinicians who might be interested in modeling the learning curve, when monitoring memory in marijuana users, the senior authors developed an MS Windows application "LCMT" to help model the learning curve from raw learning data in the California Verbal Learning Test and other similar memory tests. This app is available on request from the senior authors.

For those who want to constantly monitor the state of their memory on their own, the senior authors developed a Windows application for testing memory called "Memory Monitoring Tool". This app allows long-lasting memory monitoring and might be used by marijuana users.

\section{ACKNOWLEDGEMENTS}

Portions of this research were supported by National Science Foundation grant DBI 1560389.

\section{CONFLICTS OF INTEREST}

The authors declare no conflicts of interest regarding the publication of this manuscript.

\section{REFERENCES}

1. Abramson, C.I. and Stepanov, I.I. (2007) The Application of a Mathematical Model for the Assessment of the Memorization Curve in Drug Addicts and Alcoholics. Journal of Drug Addiction, Education and Eradication, 3 , 239-262.

2. Stepanov, I.I., Abramson, C.I., Yates, K.F. and Convit, A. (2011) The Application of a Mathematical Model to the CVLT Learning Curve for Patients with Type 2 Diabetes Mellitus. In: Berjardt, L.V., Ed., Advances in Medicine and Biology, Nova Science Publishers Inc., New York, 1-47.

3. Stepanov, I.I., Abramson, C.I., Hoogs, M. and Benedict, R.H.B. (2012) Overall Memory Impairment Identification with Mathematical Modeling of the CVLT-II Learning Curve in Multiple Sclerosis. Multiple Sclerosis International, 2012, Article ID: 312503. https://doi.org/10.1155/2012/312503

4. Stepanov, I.I., Abramson, C.I., Wolf, O.T. and Convit, A. (2010) The Application of the First Order System Transfer Function for Fitting the California Verbal Learning Test Learning Curve. Journal of the International Neuropsychological Society, 16, 443-452. https://doi.org/10.1017/S1355617709991457 
5. Stepanov, I.I., Abramson, C.I. and Warschausky, S. (2011) Assessment of the Learning Curve from the California Verbal Learning Test-Children's Version with the First-Order System Transfer Function. Child Neuropsychology, 17, 330-346. https://doi.org/10.1080/09297049.2010.541234

6. Stepanov, I.I. and Abramson, C.I. (2008) The Application of the First Order System Transfer Function for Fitting the 3-Arm Radial Maze Learning Curve. Journal of Mathematical Psychology, 52, 309-319.

https://doi.org/10.1016/j.jmp.2008.08.001

7. Abramson, C.I. and Stepanov, I.I. (2012) The Use of the First Order System Transfer Function in the Analysis of Proboscis Extension Learning of Honey Bees, Apis mellifera L., Exposed to Pesticides. Bulletin of Environmental Contamination and Toxicology, 88, 559-562. https://doi.org/10.1007/s00128-011-0512-8

8. De Stefano, L.A., Stepanov, I.I. and Abramson, C.I. (2014) The First Order Transfer Function in the Analysis of Agrochemical Data in Honey Bee (Apis mellifera L.): Proboscis Extension Reflex (PER) Studies. Insects, 5, 167-198. https://doi.org/10.3390/insects5010167

9. Delis, D.C., Kramer, J.H., Kaplan, E. and Ober, B.A. (2000) California Verbal Learning Test (2nd Edition): Adult Version Manual. The Psychological Corporation, San Antonio.

10. Zimprich, D. and Rast, P. (2009) Verbal Learning Changes in Older Adults across 18 Months. Aging, Neuropsychology, and Cognition, 16, 461-484. http://www.psypress.com/anc https://doi.org/10.1080/13825580902785663

11. Luria, A.R. (1962/1966) Higher Cortical Functions in Man. Originally Published by Moscow University Press, 1962, Translated by Haigh, B., Basic Books, New York.

12. Benedict, R. (2005) Effects of Using Same- versus Alternate-Form Memory Tests during Short-Interval Repeated Assessments in Multiple Sclerosis. Journal of the International Neuropsychological Society, 11, 727-736. https://doi.org/10.1017/S1355617705050782

13. Benedict, R.H.B. and Zgaljardic, D.J. (1998) Practice Effects during Repeated Administrations of Memory Tests with and without Alternate Forms. Journal of Clinical and Experimental Neuropsychology, 20, 339-352.

https://doi.org/10.1076/jcen.20.3.339.822

14. Stegen, S., Stepanov, I., Cookfair, D., Schwartz, E., Hojnacki, D., Weinstock-Guttman, B. and Benedict, R.H.B. (2010) Validity of the California Verbal Learning Test-II in Multiple Sclerosis. The Clinical Neuropsychologist, 24, 189-202. https://doi.org/10.1080/13854040903266910

15. Kramer, J.H., Delis, D.C. and Daniel, M. (1988) Sex Differences in Verbal Learning. Journal of Clinical Psychology, 44, 907-915. https://doi.org/10.1002/1097-4679(198811)44:6<907::AID-JCLP2270440610>3.0.CO;2-8

16. Liu, D., et al. (2014) Effects of Curcumin on Learning and Memory Deficits, BDNF, and ERK Protein Expression in Rats Exposed to Chronic Unpredictable Stress. Behavioural Brain Research, 271, 116-121. https://doi.org/10.1016/j.bbr.2014.05.068

17. Fruson, L., Dalesman, S. and Lukowiak, K. (2012) A Flavonol Present in Cocoa [(-)epicatechin] Enhances Snail Memory. Journal of Experimental Biology, 215, 3566-3576. https://doi.org/10.1242/jeb.070300

18. United States Government Accountability Office (2017) Memory Supplements, Clarifying FDA and FTC Roles Could Strengthen Oversight and Enhance Consumer Awareness: Report to Congressional Requesters. United States Government Accountability Office, Washington DC. http://link.library.mst.edu/portal/Memory-supplements-clarifying-FDA-and-FTC-roles/LtnM5Xtweo4

19. Mohamed, S., Lee Ming, T. and Jaffri, J. (2013) Cognitive Enhancement and Neuroprotection by Catechin-Rich Oil Palm Leaf Extract Supplement. Journal of the Science of Food and Agriculture, 93, 819-827. https://doi.org/10.1002/jsfa.5802 
20. Bolla, K.I., Brown, K., Eldreth, D., Tate, K. and Cadet, J.L. (2002) Dose-Related Neurocognitive Effects of Marijuana Use. Neurology, 59, 1337-1343. https://doi.org/10.1212/01.WNL.0000031422.66442.49

21. Schweinsburg, A.D., Brown, S. and Tapert, S.F. (2008) The Influence of Marijuana Use on Neurocognitive Functioning in Adolescents. Current Drug Abuse Reviews, 1, 99-111.

https://doi.org/10.2174/1874473710801010099 